TRANSACTIONS OF THE

AMERICAN MATHEMATICAL SOCIETY

Volume 350, Number 10, October 1998, Pages 4195-4220

S $0002-9947(98) 02321-6$

\title{
ON THE RIGIDITY THEOREM FOR ELLIPTIC GENERA
}

\author{
ANAND DESSAI AND RAINER JUNG
}

\begin{abstract}
We give a detailed proof of the rigidity theorem for elliptic genera. Using the Lefschetz fixed point formula we carefully analyze the relation between the characteristic power series defining the elliptic genera and the equivariant elliptic genera. We show that equivariant elliptic genera converge to Jacobi functions which are holomorphic. This implies the rigidity of elliptic genera. Our approach can be easily modified to give a proof of the rigidity theorem for the elliptic genera of level $N$.
\end{abstract}

\section{INTRODUCTION}

In this article we give a detailed proof of the rigidity theorem for elliptic genera of Spin-manifolds with a special focus on the function theory involved. Elliptic genera were introduced by Ochanine in [Oc87] (see also [LaSt88]). Witten gave two descriptions of elliptic genera as power series of indices of twisted Dirac operators ([Wi86]). He conjectured the rigidity theorem which states that these elliptic genera are rigid for $S^{1}$-actions on Spin-manifolds (for a precise statement see Theorem 2.5). In the semi-free case Ochanine gave a proof of the rigidity theorem in [Oc86]. The general case was proven by Taubes [Ta89] using a Dirac operator on the normal bundle to the embedding of the manifold into its loop space and by Bott-Taubes [BoTa89] using elliptic function theory. Later Liu observed that the rigidity theorem is equivalent to the holomorphicity of an associated Jacobi function (cf. [Li92] and [Li95]; for an introduction to Jacobi forms we refer to [EiZa85]). By exploring the modularity properties of this Jacobi function Liu gave a simplified proof avoiding the complicated technical transfer argument which was used in [BoTa89]. For the history of elliptic genera we refer to [La86].

In the proofs of Bott-Taubes and Liu the parts dealing with function theory and with higher dimensional fixed point components were rather short. So several people suggested that a detailed proof of the rigidity theorem should be carried out. More precisely this proof should focus on the following steps

- equivariant elliptic genera as $q$-power series converge to meromorphic functions,

- these meromorphic functions are holomorphic,

- these functions are Jacobi functions of index 0,

and give details also for the case of higher dimensional fixed point components. The purpose of this article is to carry out this proof. Most of the material evolved from

Received by the editors January 7, 1997.

1991 Mathematics Subject Classification. Primary 58G10, 19L47, 11F03; Secondary 57S15, $13 \mathrm{~N} 10$.

Key words and phrases. Rigidity theorem, elliptic genera, index theory, Jacobi functions.

(c)1998 American Mathematical Society 
discussions of both authors with Th. Berger. From Liu (cf. [Li92]) we borrowed the beautiful idea of using Jacobi functions.

We present a framework which is also useful to study other rigidity phenomena, in particular in the case of genera. For stable almost complex manifolds Hirzebruch proved the rigidity of level $N$ elliptic genera (cf. [Hi88], see also [HiBeJu92], Appendix III) also conjectured by Witten (cf. [Wi86]). Our approach can be easily modified to give a more detailed account of this theorem. In [De96] the rigidity theorems for the elliptic genera and level $N$ elliptic genera have been extended to various twisted versions for $S_{p i n}{ }^{c}$-manifolds. In order to present the basic ideas most clearly we decided to restrict ourselves to the case of the classical elliptic genera. For the proof of more general rigidity theorems we refer the reader to [De96].

The outline of the proof is as follows: In the first chapter we study the $S^{1}$ equivariant index of a twisted Dirac-operator on a Spin-manifold $M$. The Lefschetz fixed point formula gives a description of the equivariant index in terms of cohomological data (cf. Theorem 2.8). Now we assume that the twisted operator comes from an exponential representation $V$ of the Spin-group (cf. Definition 2.15, Part 1). So it also defines a genus, i.e. a multiplicative invariant on the bordism ring. In this case we define a so-called building block associated to the exponential representation $V$, which is closely related to the characteristic power series defining the genus in the Hirzebruch formalism (cf. Definition 2.15, Part 2). Then the equivariant index can be expressed in terms of the building block and the data of the $S^{1}$-action over the fixed point components. More precisely we show that it is given by evaluating a polynomial $\mathfrak{P}_{M}$ in basic differential operators on the building block (cf. Proposition 2.17, Part 2). The polynomial $\mathfrak{P}_{M}$ only depends on the operation of $S^{1}$ over the fixed point components and not on the twisted Dirac-operator itself. This result leads to the definition of DSC-rings (cf. Definition 2.18): They are rings on which such polynomials $\mathfrak{P}_{M}$ act in a graded way. So whenever the building block is in some DSC-ring, the equivariant index will also be in this ring (cf. Proposition 2.20). In this way properties of the building block can be carried over to the equivariant index.

In the second chapter we apply this principle to DSC-rings which are suitable to study elliptic genera. Using the meromorphicity and modularity properties of the building blocks associated to elliptic genera, we show that the equivariant elliptic genera (i.e. the equivariant indices) extend to special Jacobi functions of index 0 (cf. Proposition 3.26, Part 1). From a proposition of Th. Berger it follows that these Jacobi functions have no poles on $S^{1}$ (cf. Proposition 3.10 and Corollary 3.17). For the next step it is necessary to use both of the two different parametrizations of elliptic genera (as given by Witten). The two parametrizations are an orbit of the action of $S L_{2}(\mathbb{Z})$ on Jacobi functions (for a more precise statement see Proposition 3.26, Part 2). Since both have no poles on $S^{1}$, we can use the transformation properties of Jacobi functions to show that the meromorphic Jacobi functions to which the equivariant elliptic genera extend are actually holomorphic. Since any such function is constant (in the variable $z$ ) this proves the rigidity theorem (cf. Theorem 3.29).

\section{Cohomological Considerations}

2.1. Elliptic Genera. In this article (unless stated otherwise) all manifolds are assumed to be smooth, closed and oriented. In this section we recall the construction 
of elliptic genera for Spin-manifolds as power series of indices of certain differential operators (cf. [Wi86]).

Let $M$ be a $2 m$-dimensional manifold with $\operatorname{Spin}$-structure given by a $\operatorname{Spin}(2 m)$ principal bundle $P \rightarrow M$ together with an isomorphism $P \times_{\operatorname{Spin}(2 m)} \mathbb{R}^{2 m} \cong T M$ of oriented vector bundles, where $T M$ denotes the tangent bundle of $M$. Let $\rho: \operatorname{Spin}(2 m) \rightarrow S O(2 m)$ be the covering map and $\rho^{*}: R(S O(2 m)) \rightarrow R(\operatorname{Spin}(2 m))$ be the induced injection of the complex representation rings. In the following a complex $S O(2 m)$-representation $V$ will always be identified with $\rho^{*}(V)$. To any complex $\operatorname{Spin}(2 m)$-representation $V$ one can assign the associated complex vector bundle $P \times{ }_{\operatorname{Spin}(2 m)} V$ over $M$. This defines a ring homomorphism

$$
R(\operatorname{Spin}(2 m)) \rightarrow K(M) .
$$

Since $M$ is spin one can construct for $E \in K(M)$ a twisted Dirac operator $D(M ; E)$ with a well-defined index denoted by $\hat{A}(M ; E)$. This gives a $\mathbb{Z}$-module homomorphism

$$
\hat{A}(M ; \cdot): K(M) \rightarrow \mathbb{Z}, E \mapsto \hat{A}(M ; E) .
$$

For $E \in K(M)$ one can also construct a twisted signature operator $d(M ; E)$ with a well-defined index denoted by $\operatorname{sign}(M ; E)$. Again this gives a $\mathbb{Z}$-module homomorphism

$$
\operatorname{sign}(M ; \cdot): K(M) \rightarrow \mathbb{Z}, E \mapsto \operatorname{sign}(M ; E) .
$$

If the vector bundle $E$ is associated to $P$ by a representation $V$ of $\operatorname{Spin}(2 m)$ we will also use the notation $\hat{A}(M ; V)$ and $\operatorname{sign}(M ; V)$ instead of $\hat{A}(M ; E)$ and $\operatorname{sign}(M ; E)$, respectively. For $V=1$ these are the classical genera $\hat{A}(M)=\hat{A}(M ; 1)$ and $\operatorname{sign}(M)=\operatorname{sign}(M ; 1)$.

We denote the complex half-spin representations of $\operatorname{Spin}(2 \mathrm{~m})$ by $\triangle^{+}$and $\triangle^{-}$, and the associated vector bundles $P \times_{\operatorname{Spin}(2 m)} \triangle^{ \pm}$by $\triangle_{P}^{ \pm}$.

Remark 2.1. For a Spin-manifold $M$ the index $\operatorname{sign}(M ; E)$ is equal to the index of a twisted Dirac operator:

$$
\operatorname{sign}(M ; E)=\hat{A}\left(M ; E \otimes\left(\triangle_{P}^{+}+\triangle_{P}^{-}\right)\right) .
$$

Analogously we can consider the homomorphism $R(\operatorname{Spin}(2 m))[[q]] \rightarrow K(M)[[q]]$ of $\mathbb{Z}[[q]]$-algebras and the $\mathbb{Z}[[q]]$-module homomorphisms

and

$$
K(M)[[q]] \rightarrow \mathbb{Z}[[q]], \sum_{n=0}^{\infty} E_{n} q^{n} \mapsto \sum_{n=0}^{\infty} \hat{A}\left(M ; E_{n}\right) q^{n}
$$

$$
K(M)[[q]] \rightarrow \mathbb{Z}[[q]], \sum_{n=0}^{\infty} E_{n} q^{n} \mapsto \sum_{n=0}^{\infty} \operatorname{sign}\left(M ; E_{n}\right) q^{n} .
$$

Now let $S^{i}$ and $\Lambda^{i}$ be the usual symmetric and exterior power representations in the complex representation ring of $S O(2 m)$ and let $S_{t}$ and $\Lambda_{t}$ be given by

$$
S_{t}=\sum_{i=0}^{\infty} S^{i} t^{i}, \Lambda_{t}=\sum_{i=0}^{2 m} \Lambda^{i} t^{i} \in R(S O(2 m))[[t]] .
$$

Following Witten (cf. [Wi86]) we now define the elliptic genera using two power series of representations $R$ and $R^{0}$. 
Definition 2.2. 1. Let

$$
R=\sum_{n=0}^{\infty} R_{n} q^{n}:=\left(\triangle^{+}+\triangle^{-}\right) \otimes \bigotimes_{n=1}^{\infty} S_{q^{n}} \otimes \bigotimes_{n=1}^{\infty} \Lambda_{q^{n}} \otimes C \in R(\operatorname{Spin}(2 m))[[q]]
$$

and

$$
R^{0}=\sum_{n=0}^{\infty} R_{n}^{0} q^{n}:=\bigotimes_{n=1}^{\infty} S_{q^{2 n}} \otimes \bigotimes_{n=1}^{\infty} \Lambda_{-q^{2 n-1}} \otimes C^{0} \in R(S O(2 m))[[q]],
$$

where

$$
C:=\prod_{n=1}^{\infty} \frac{\left(1-q^{n}\right)^{2 m}}{\left(1+q^{n}\right)^{2 m}} \quad \text { and } \quad C^{0}:=\prod_{n=1}^{\infty} \frac{\left(1-q^{2 n}\right)^{2 m}}{\left(1-q^{2 n-1}\right)^{2 m}} .
$$

2. For any $2 m$-dimensional Spin-manifold $M$ we define the elliptic genus $\varphi(M)$ of $M$ at the cusp $i \infty$ and the elliptic genus $\varphi^{0}(M)$ of $M$ at the cusp 0 by $^{1}$

$$
\varphi(M):=\hat{A}(M ; R)=\sum_{n=0}^{\infty} \hat{A}\left(M ; R_{n}\right) q^{n} \in \mathbb{Z}[[q]]
$$

and

$$
\varphi^{0}(M):=\hat{A}\left(M ; R^{0}\right)=\sum_{n=0}^{\infty} \hat{A}\left(M ; R_{n}^{0}\right) q^{n} \in \mathbb{Z}[[q]] .
$$

The elliptic genus of $M$ at the cusp $i \infty$ may be written as a power series of indices of twisted signatures (cf. Remark 2.1):

$$
\varphi(M)=\operatorname{sign}\left(M ; \bigotimes_{n=1}^{\infty} S_{q^{n}} \otimes \bigotimes_{n=1}^{\infty} \Lambda_{q^{n}}\right) \cdot C \in \mathbb{Z}[[q]] .
$$

The first terms in these power series are

$$
\begin{aligned}
\varphi(M) \equiv(\operatorname{sign}(M) & +2 \operatorname{sign}\left(M ; \Lambda^{1}\right) q \\
& \left.+\operatorname{sign}\left(M ; 2 \Lambda^{1}+S^{2}+\left(\Lambda^{1}\right)^{2}+\Lambda^{2}\right) q^{2}\right) \cdot C \bmod \left(q^{3}\right)
\end{aligned}
$$

and

$$
\varphi^{0}(M) \equiv\left(\hat{A}(M)-\hat{A}\left(M ; \Lambda^{1}\right) q+\hat{A}\left(M ; \Lambda^{2}+\Lambda^{1}\right) q^{2}\right) \cdot C^{0} \bmod \left(q^{3}\right) .
$$

In Proposition 3.26 we will see that the two expressions $\varphi(M)$ and $\varphi^{0}(M)$ are two different descriptions of the same invariant of $M$.

2.2. Equivariant Elliptic Genera and the Rigidity Theorem. In this section we define equivariant elliptic genera for $S^{1}$-actions and state the rigidity theorem. We assume that $M$ carries a smooth $S^{1}$-action preserving its $S p i n$-structure, i.e. $P \rightarrow M$ is an $S^{1}$-equivariant $\operatorname{Spin}(2 m)$-principal bundle. Observe that any given $S^{1}$-action preserves the Spin-structure after doubling the action using the double cover $S^{1} \rightarrow S^{1}, \lambda \mapsto \lambda^{2}$.

Let $E$ be an $S^{1}$-equivariant complex vector bundle over $M$. Then the indices $\hat{A}(M ; E)$ and $\operatorname{sign}(M ; E)$ refine to well-defined equivariant indices

$$
\hat{A}_{S^{1}}(M ; E) \in R\left(S^{1}\right) \quad \text { and } \operatorname{sign}_{S^{1}}(M ; E) \in R\left(S^{1}\right) .
$$

\footnotetext{
${ }^{1}$ This differs from the definition of $\varphi(M)$ in [HiBeJu92] by a factor of $2^{m}$.
} 
This induces homomorphisms of $R\left(S^{1}\right)$-modules from $K_{S^{1}}(M)$ to $R\left(S^{1}\right)$, such that for the corresponding characters we have

$$
\hat{A}_{S^{1}}(M ; E)(1)=\hat{A}(M ; E) \text { and } \operatorname{sign}_{S^{1}}(M ; E)(1)=\operatorname{sign}(M ; E) .
$$

Here (and in the following) we make no distinction between representations of $S^{1}$ and their corresponding characters. As in the previous section we extend the definition and notation to $V \in R(\operatorname{Spin}(2 m))$ and power series in $q$. Note that the $S^{1}$-action on the principal bundle $P$ induces an $S^{1}$-action on any associated vector bundle.

Remark 2.3. For a Spin-manifold $M$ with $S^{1}$-action preserving the Spin-structure the equivariant index $\operatorname{sign}_{S^{1}}(M ; E)$ is equal to the equivariant index $\hat{A}_{S^{1}}(M ; E \otimes$ $\left.\left(\triangle_{P}^{+}+\triangle_{P}^{-}\right)\right)$of a twisted Dirac operator.

Definition 2.4. For any $2 m$-dimensional Spin-manifold $M$ with Spin-preserving $S^{1}$-action we define the equivariant elliptic genera at the cusps $i \infty$ and 0 by

and

$$
\varphi_{S^{1}}(M):=\hat{A}_{S^{1}}(M ; R)=\sum_{n=0}^{\infty} \hat{A}_{S^{1}}\left(M ; R_{n}\right) q^{n} \in R\left(S^{1}\right)[[q]]
$$

$$
\varphi_{S^{1}}^{0}(M):=\hat{A}_{S^{1}}\left(M ; R^{0}\right)=\sum_{n=0}^{\infty} \hat{A}_{S^{1}}\left(M ; R_{n}^{0}\right) q^{n} \in R\left(S^{1}\right)[[q]] .
$$

Note that $\varphi_{S^{1}}(M)(1)=\varphi(M)$ and $\varphi_{S^{1}}^{0}(M)(1)=\varphi^{0}(M)$. By Remark 2.3 the equivariant elliptic genus $\varphi_{S^{1}}(M)$ is equal to

$$
\varphi_{S^{1}}(M)=\operatorname{sign}_{S^{1}}\left(M ; \bigotimes_{n=1}^{\infty} S_{q^{n}} \otimes \bigotimes_{n=1}^{\infty} \Lambda_{q^{n}}\right) \cdot C \in R\left(S^{1}\right)[[q]] .
$$

Now we are in the position to state the rigidity theorem.

Theorem 2.5 (Rigidity Theorem [Wi86], [Ta89], [BoTa89], [Li92]). Let $M$ be a $2 m$-dimensional Spin-manifold with Spin-preserving $S^{1}$-action. Then the equivariant elliptic genera $\varphi_{S^{1}}(M)$ and $\varphi_{S^{1}}^{0}(M)$ are both rigid, i.e. they are power series in $q$ with coefficients which are constant as characters of $S^{1}$. In other words

$$
\varphi_{S^{1}}(M)=\varphi(M) \quad \text { and } \quad \varphi_{S^{1}}^{0}(M)=\varphi^{0}(M) .
$$

Remark 2.6. Note that the identity in Theorem 2.5 is an identity of power series in $q$, i.e.

$$
\hat{A}_{S^{1}}\left(M ; R_{n}\right)=\hat{A}\left(M ; R_{n}\right) \quad \text { and } \quad \hat{A}_{S^{1}}\left(M ; R_{n}^{0}\right)=\hat{A}\left(M ; R_{n}^{0}\right)
$$

for all $n \geq 0$. So the theorem states that all the equivariant indices $\hat{A}_{S^{1}}\left(M ; R_{n}\right)$ and $\hat{A}_{S^{1}}\left(M ; R_{n}^{0}\right)$ are in fact constant as characters on $S^{1}$.

2.3. Cohomological Formulas for Indices. In this section we recall the cohomological formulas for elliptic genera in the non-equivariant case.

Let $\left\{ \pm x_{i}\right\}$ be the roots of $T M$, thus $\prod_{i=1}^{m}\left(1+x_{i}^{2}\right)=p(M)=\sum_{i=0}^{m} p_{i}(M)$ is the total Pontrjagin class of $M$. From the Atiyah-Singer Index Theorem (cf. [AtSiIII68]) one gets an explicit formula for $\hat{A}(M ; E)$ in terms of cohomological data:

$$
\hat{A}(M ; E)=(-1)^{m}\left\langle\prod_{i=1}^{m}\left(x_{i} \cdot \frac{1}{e^{\frac{x_{i}}{2}}-e^{-\frac{x_{i}}{2}}}\right) \cdot \operatorname{ch}(E),[M]\right\rangle,
$$


where $c h: K(M) \rightarrow H^{*}(M ; \mathbb{Q})$ is the usual Chern character ring homomorphism, $[M]$ is the fundamental cycle of the oriented manifold $M$ and $\langle\cdot, \cdot\rangle$ is the Kronecker pairing between homology and cohomology.

Note that if $V$ is a $\operatorname{Spin}(2 m)$-representation of rank $r$ with weights ${ }^{2} \omega_{i}\left(z_{1}, \ldots, z_{m}\right)$, $i=1, \ldots, r$,

$$
\omega_{i}\left(z_{1}, \ldots, z_{m}\right)=\sum_{j=1}^{m} \omega_{i}^{j} z_{j}, \omega_{i}^{j} \in \frac{1}{2} \cdot \mathbb{Z}
$$

then

$$
\operatorname{ch}\left(P \times_{\operatorname{Spin}(2 m)} V\right)=\sum_{i=1}^{r} e^{\omega_{i}\left(x_{1}, \ldots, x_{m}\right)} .
$$

We will also use the shorthand notation $\operatorname{ch}(V)$ for $\operatorname{ch}\left(P \times_{\operatorname{Spin}(2 m)} V\right)$.

Since $c h$ is a ring homomorphism its canonical extension

$$
\operatorname{ch}: K(M)[[q]] \rightarrow H^{*}(M ; \mathbb{Q})[[q]]
$$

is a $\mathbb{Z}[[q]]$-algebra homomorphism. If $V=\sum_{n=0}^{\infty} V_{n} q^{n} \in R(\operatorname{Spin}(2 m))[[q]]$ is a power series of representations, where $V_{n}$ has rank $r(n)$ and weights $\omega_{n, i}\left(z_{1}, \ldots, z_{m}\right)=$ $\sum_{j=1}^{m} \omega_{n, i}^{j} z_{j}$ for $i=1, \ldots, r(n)$, then the corresponding Chern character is equal to

$$
\operatorname{ch}(V)=\sum_{n=0}^{\infty} \sum_{i=1}^{r(n)} e^{\omega_{n, i}\left(x_{1}, \ldots, x_{m}\right)} \cdot q^{n}
$$

Definition 2.7. 1. Let $F_{1}\left(\mu_{1}, \ldots, \mu_{m}\right):=\prod_{i=1}^{m} \frac{1}{\mu_{i}^{\frac{1}{2}}-\mu_{i}^{-\frac{1}{2}}} \in \mathbb{Q}\left(\mu_{1}^{\frac{1}{2}}, \ldots, \mu_{m}^{\frac{1}{2}}\right)$.

2. For a power series of representations $V=\sum_{n=0}^{\infty} V_{n} q^{n} \in R(\operatorname{Spin}(2 m))[[q]]$ with weights $\omega_{n, i}$ as in (2) we define its formal Chern character

$$
F_{2, V}\left(\mu_{1}, \ldots, \mu_{m}\right):=\sum_{n=0}^{\infty} \sum_{i=1}^{r(n)} e^{\omega_{n, i}\left(z_{1}, \ldots, z_{m}\right)} q^{n} \in \mathbb{Q}\left[\mu_{1}^{ \pm \frac{1}{2}}, \ldots, \mu_{m}^{ \pm \frac{1}{2}}\right][[q]]
$$

where $\mu_{j}=e^{z_{j}}$ for $j=1, \ldots, m$.

Using the extension of the Chern character to power series and the previous definition we can restate formula (1), giving the non-equivariant index of a twisted Dirac operator, in the following way:

$$
\hat{A}(M ; V)=(-1)^{m}\left\langle\left(\prod_{i=1}^{m} x_{i}\right) \cdot F_{1}\left(e^{x_{1}}, \ldots, e^{x_{m}}\right) \cdot F_{2, V}\left(e^{x_{1}}, \ldots, e^{x_{m}}\right),[M]\right\rangle .
$$

Now we specialize formula (3) to the case of elliptic genera. Consider the wellknown formulas

$$
\begin{aligned}
\operatorname{ch}\left(S_{q}\right) & =\prod_{i=1}^{m} \frac{1}{\left(1-q e^{x_{i}}\right)\left(1-q e^{-x_{i}}\right)}, \\
\operatorname{ch}\left(\Lambda_{q}\right) & =\prod_{i=1}^{m}\left(1+q e^{x_{i}}\right)\left(1+q e^{-x_{i}}\right)
\end{aligned}
$$

\footnotetext{
${ }^{2}$ As usual we give weights of $\operatorname{Spin}(2 m)$-representations in terms of $S O(2 m)$.
} 
and

$$
\operatorname{ch}\left(\triangle^{+}+\triangle^{-}\right)=\prod_{i=1}^{m}\left(e^{\frac{x_{i}}{2}}+e^{-\frac{x_{i}}{2}}\right) .
$$

Then the Chern characters of the power series of representations $R$ and $R^{0}$ in $R(\operatorname{Spin}(2 m))[[q]]$ given in Definition 2.2 are equal to

and

$$
\operatorname{ch}(R)=\prod_{i=1}^{m}\left(\left(e^{\frac{x_{i}}{2}}+e^{-\frac{x_{i}}{2}}\right) \prod_{n=1}^{\infty} \frac{\left(1+q^{n} e^{x_{i}}\right)\left(1+q^{n} e^{-x_{i}}\right)\left(1-q^{n}\right)^{2}}{\left(1-q^{n} e^{x_{i}}\right)\left(1-q^{n} e^{-x_{i}}\right)\left(1+q^{n}\right)^{2}}\right)
$$

$$
\operatorname{ch}\left(R^{0}\right)=\prod_{i=1}^{m}\left(\prod_{n=1}^{\infty} \frac{\left(1-q^{2 n-1} e^{x_{i}}\right)\left(1-q^{2 n-1} e^{-x_{i}}\right)\left(1-q^{2 n}\right)^{2}}{\left(1-q^{2 n} e^{x_{i}}\right)\left(1-q^{2 n} e^{-x_{i}}\right)\left(1-q^{2 n-1}\right)^{2}} .\right.
$$

So the formal Chern characters $F_{2, R}$ and $F_{2, R^{0}}$ of $R$ and $R^{0}$ are equal to

$$
F_{2, R}\left(\mu_{1}, \ldots, \mu_{m}\right)=\prod_{i=1}^{m}\left(\left(\mu_{i}^{\frac{1}{2}}+\mu_{i}^{-\frac{1}{2}}\right) \prod_{n=1}^{\infty} \frac{\left(1+q^{n} \mu_{i}\right)\left(1+q^{n} \mu_{i}^{-1}\right)\left(1-q^{n}\right)^{2}}{\left(1-q^{n} \mu_{i}\right)\left(1-q^{n} \mu_{i}^{-1}\right)\left(1+q^{n}\right)^{2}}\right)
$$

and

$$
F_{2, R^{0}}\left(\mu_{1}, \ldots, \mu_{m}\right)=\prod_{i=1}^{m}\left(\prod_{n=1}^{\infty} \frac{\left(1-q^{2 n-1} \mu_{i}\right)\left(1-q^{2 n-1} \mu_{i}^{-1}\right)\left(1-q^{2 n}\right)^{2}}{\left(1-q^{2 n} \mu_{i}\right)\left(1-q^{2 n} \mu_{i}^{-1}\right)\left(1-q^{2 n-1}\right)^{2}}\right) .
$$

Applying formula (3) to the elliptic genera we get

$$
\varphi(M)=(-1)^{m}\left\langle\prod_{i=1}^{m}\left(x_{i} \cdot \frac{e^{x_{i}}+1}{e^{x_{i}}-1} \cdot \prod_{n=1}^{\infty} \frac{\left(1+q^{n} e^{x_{i}}\right)\left(1+q^{n} e^{-x_{i}}\right)\left(1-q^{n}\right)^{2}}{\left(1-q^{n} e^{x_{i}}\right)\left(1-q^{n} e^{-x_{i}}\right)\left(1+q^{n}\right)^{2}}\right),[M]\right\rangle
$$

and

$$
\begin{aligned}
& \varphi^{0}(M)= \\
& (-1)^{m}\left\langle\prod_{i=1}^{m}\left(\frac{x_{i}}{e^{\frac{x_{i}}{2}}-e^{-\frac{x_{i}}{2}}} \cdot \prod_{n=1}^{\infty} \frac{\left(1-q^{2 n-1} e^{x_{i}}\right)\left(1-q^{2 n-1} e^{-x_{i}}\right)\left(1-q^{2 n}\right)^{2}}{\left(1-q^{2 n} e^{x_{i}}\right)\left(1-q^{2 n} e^{-x_{i}}\right)\left(1-q^{2 n-1}\right)^{2}}\right),[M]\right\rangle,
\end{aligned}
$$

where both expressions are in $\mathbb{Q}[[q]]$. In fact from the earlier definition as indices we know that the expressions are in $\mathbb{Z}[[q]]$.

2.4. Cohomological Formulas for Equivariant Indices. In this section we give the cohomological version of the Lefschetz fixed point formula for equivariant indices (cf. [AtSiIII68]). It describes the global equivariant index in terms of local invariants associated to the $S^{1}$-action over the fixed point components of the manifold.

Let $M$ be a $2 m$-dimensional Spin-manifold with Spin-preserving $S^{1}$-action. Let $M^{S^{1}} \subset M$ be the fixed point manifold of the $S^{1}$-action on $M$ and let $Y$ be a connected component of $M^{S^{1}}$. Since $Y$ is a trivial $S^{1}$-space, the tangent bundle $T M$ of $M$ restricted to $Y$ splits equivariantly as a direct sum of the tangent bundle $\nu_{0}$ of $Y$ and the normal bundle $\nu$ of $Y$ in $M$. The latter splits equivariantly as a finite direct sum $\nu=\bigoplus_{k=1}^{l} \nu_{k}$, where the non-trivial $S^{1}$-action on $\nu_{k}$ induces a complex structure on $\nu_{k}$, s.t. $\lambda \in S^{1}$ acts on $\nu_{k}$ by multiplication with $\lambda^{m_{Y}^{k}}$ and all $m_{Y}^{k}$ are positive integers. These integers will be called rotation numbers of the $S^{1}$-action at $Y$. We define $m_{Y}^{0}:=0$ which we interpret as the tangential rotation number of $Y$. Let the orientation of $Y$ be induced by the orientation of $M$ and the complex structures on $\nu_{k}$. We denote by $r(k)$ the complex rank of $\nu_{k}$ and let $r(0)$ be equal to half of the dimension of $Y$. We remark that the integers $l$ and $r(k)$ for 
$k=0, \ldots, l$, depend on the chosen component $Y$. We are now in the position to state the Lefschetz fixed point theorem.

Theorem 2.8 (Lefschetz fixed point formula [AtSiIII68]). Let $M$ be a 2m-dimensional Spin-manifold with Spin-preserving $S^{1}$-action and let $\mathcal{Y}$ be the set of connected components of $M^{S^{1}}$. Let $V \in R(\operatorname{Spin}(2 m))[[q]]$. Then

$$
\hat{A}_{S^{1}}(M ; V)=\sum_{Y \in \mathcal{Y}} a(Y, V),
$$

where $a(Y, V) \in \mathbb{Q}\left(\lambda^{\frac{1}{2}}\right)[[q]]$ is given by

$$
\begin{aligned}
a(Y, V)(\lambda)=(-1)^{m}\left\langle\left(\prod_{j=1}^{r(0)} x_{0, j}\right) \cdot F_{1}\left(e^{x_{0,1}+m_{Y}^{0} \cdot z}, \ldots, e^{x_{k, j}+m_{Y}^{k} \cdot z}, \ldots, e^{x_{l, r(l)}+m_{Y}^{l} \cdot z}\right)\right. \\
\\
\left.\cdot F_{2, V}\left(e^{x_{0,1}+m_{Y}^{0} \cdot z}, \ldots, e^{x_{k, j}+m_{Y}^{k} \cdot z}, \ldots, e^{x_{l, r(l)}+m_{Y}^{l} \cdot z}\right),[Y]\right\rangle
\end{aligned}
$$

for $\lambda=e^{z}$. Here $m_{Y}^{k}$ are the rotation numbers of $Y$ and $x_{k, j}$ are the roots of the bundle $v_{k}$.

Remark 2.9. 1. Recipe: The formula giving $a(Y, V)$ is obtained from formula (3) for the non-equivariant index of $M$ by replacing the Euler class $\prod_{i=1}^{m} x_{i}$ of $M$ by the Euler class $\prod_{j=1}^{r(0)} x_{0, j}$ of $Y$ and replacing the roots $x_{i}$ of $T M$ by $x_{k, j}+m_{Y}^{k} \cdot z$.

2. By definition $\hat{A}_{S^{1}}(M ; V)$ is a power series in $q$ with coefficients which are characters on $S^{1}$, i.e. Laurent polynomials in $\lambda$. The given formula describes the global equivariant index $\hat{A}_{S^{1}}(M ; V)$ in terms of local invariants $a(Y, V)$ associated to the fixed point components $Y$ of $M$. We will see in the next section (cf. Theorem 2.13) that each local invariant $a(Y, V)$ is an element in $\mathbb{Q}\left(\lambda^{\frac{1}{2}}\right)[[q]]$, where the coefficients of this power series might have poles only at zero or at any $\lambda$ with $\lambda^{m_{Y}^{k}}=1$. Identity (4) of the Lefschetz fixed point theorem is proven for topological generators $\lambda$ of $S^{1}$. Since the set of topological generators is dense on $S^{1}$, identity (4) is $q$-coefficientwise an identity of rational functions. Since each coefficient on the left side is actually a Laurent polynomial in $\lambda$, the possible poles on $S^{1}$ on the right side must cancel out by summation over the fixed point components $Y$.

2.5. The Lefschetz Fixed Point Formula - Revisited. In this section we will have a closer look at the Lefschetz fixed point formula. We will define a power series $G_{V, \mathcal{N}(Y)}$ which is closely related to an equivariant analog of the formal Chern character of $V$. Then we will rephrase the evaluation of the cohomological expression on the fundamental class to express the local invariants $a(Y, V)$ as a $\mathbb{Q}$-linear combination of the coefficients of $G_{V, \mathcal{N}(Y)}$. The coefficients of this $\mathbb{Q}$-linear combination only depend on the $S^{1}$-action over the fixed point component and not on the chosen power series of representations $V$.

Let $M$ be a $2 m$-dimensional $S p i n$-manifold with Spin-preserving $S^{1}$-action and let $Y$ be a connected component of $M^{S^{1}}$ with rotation numbers $m_{Y}^{k}$ and roots $x_{k, j}$, $j=1, \ldots, r(k)$, of the vector bundle $\nu_{k}, k=0, \ldots, l$, defined in the beginning of Section 2.4. Finally let $C(Y):=\{(k, j) \mid 0 \leq k \leq l, 1 \leq j \leq r(k)\}$ be the index set of the roots $x_{k, j}$. 
For $\mathcal{A}:=\mathbb{Q}\left(\lambda^{\frac{1}{2}}\right)[[q]]$ we study the following map: ${ }^{3}$

$\mathcal{A}\left[\left[c_{k, i}, p_{h}, e \mid(k, i) \in C(Y), k \neq 0, h=1, \ldots, r(0)-1\right]\right] \stackrel{\iota}{\rightarrow} \mathcal{A}\left[\left[x_{k, j} \mid(k, j) \in C(Y)\right]\right]$,

where each $c_{k, i}$ is mapped by $\iota$ to the $i$-th elementary symmetric function in the $x_{k, j}$ for $k \neq 0, p_{h}$ is mapped to the $h$-th elementary symmetric function in the $x_{0, j}^{2}$, and $e$ is mapped to the product of the $x_{0, j}$, i.e.

$$
\iota\left(c_{k, i}\right)=\sigma_{i}\left(x_{k, j}\right), \quad \iota\left(p_{h}\right)=\sigma_{h}\left(x_{0, j}^{2}\right) \quad \text { and } \quad \iota(e)=\prod_{j=1}^{r(0)} x_{0, j}
$$

Note that after giving $x_{k, j}, c_{k, i}, p_{h}$ and $e$ degrees $2,2 i, 4 h$ and $2 r(0)$, respectively, the above rings become graded rings and $\iota$ a graded homomorphism.

Consider the group action $W$ on $\mathcal{A}\left[\left[x_{k, j} \mid(k, j) \in C(Y)\right]\right]$ generated by all permutations of $x_{k, 1}, \ldots, x_{k, r(k)}$ for fixed $k \in\{0, \ldots, l\}$ and even numbers of sign changes on $x_{0,1}, \ldots, x_{0, r(0)}$. Note that the image of $\iota$ lies in the invariants of the group action $W$.

Lemma 2.10. 1. The map $\iota$ is injective.

2. The image of $\iota$ is equal to the invariants of the group action $W$.

3. For every $a \in \mathcal{A}\left[\left[c_{k, i}, p_{h}, e \mid(k, i) \in C(Y), k \neq 0, h=1, \ldots, r(0)-1\right]\right]$ the coefficients of a are given by unique $\mathbb{Q}$-linear combinations of the coefficients of the monomials $\prod_{(k, j) \in C(Y)} x_{k, j}^{n_{k, j}}$ in $\iota(a)$ where $n_{k, j} \geq n_{k, j+1}$. The coefficients of these $\mathbb{Q}$-linear combinations do not depend on a.

Proof. Statements (1) and (2) follow easily from standard facts about symmetric functions after splitting domain and range of $\iota$ into appropriate tensor products. For (3) we may assume that $a$ is homogeneous of some degree $d$. Note that since $\iota$ is injective the image of $\iota$ is again a free $\mathcal{A}$-module with basis in degree $d$ given by the image of monomials in $c_{k, i}, p_{h}$ and $e$ of degree $d$. Two monomials in the $x_{k, j}$ are called equivalent iff they are in the same orbit with respect to the action of $W$. For any monomial $y$ let $\bar{y}$ denote the sum of all monomials which are equivalent to $y$. Then the equivalence classes $\prod_{(k, j) \in C(Y)} x_{k, j}^{n_{k, j}}$ for $n_{k, j} \geq n_{k, j+1}$ and $\sum_{(k, j) \in C(Y)} 2 n_{k, j}=d$ define another basis. Since the matrix for the basis change has rational entries statement (3) follows.

Recall the formulas for $F_{1}$ and $F_{2, V}$ from Definition 2.7 .

Definition 2.11. For any $m$-tuple $\mathcal{N}$ of integer numbers $m_{i}$ and any power series of representations $V$ we define

$$
\begin{aligned}
G_{1, \mathcal{N}}\left(\lambda, x_{1}, \ldots, x_{m}\right) & :=F_{1}\left(\lambda^{m_{1}} e^{x_{1}}, \ldots, \lambda^{m_{m}} e^{x_{m}}\right), \\
G_{2, V, \mathcal{N}}\left(\lambda, x_{1}, \ldots, x_{m}\right) & :=F_{2, V}\left(\lambda^{m_{1}} e^{x_{1}}, \ldots, \lambda^{m_{m}} e^{x_{m}}\right) .
\end{aligned}
$$

\footnotetext{
${ }^{3}$ The structure group of the bundle $\left.T M\right|_{Y}$ restricts to the product $G$ of $U(r(k))$ for $k \neq 0$ and $S O(2 r(0))$. The inclusion of the maximal torus in $G$ induces a map between classifying spaces which is given in cohomology with coefficients in $\mathcal{A}$ by $\iota$.
} 
For the $m$-tuple $\mathcal{N}=\mathcal{N}(Y)$ of rotation numbers $m_{k, j}:=m_{Y}^{k},(k, j) \in C(Y)$, of the fixed point component $Y$ we define

$$
\begin{gathered}
G_{V, \mathcal{N}(Y)}\left(\lambda, x_{0,1}, \ldots, x_{k, j}, \ldots, x_{l, r(l)}\right) \\
:=\left(\prod_{j=1}^{r(0)} x_{0, j}\right) \cdot G_{1, \mathcal{N}(Y)}\left(\lambda, x_{0,1}, \ldots, x_{k, j}, \ldots, x_{l, r(l)}\right) \\
\cdot G_{2, V, \mathcal{N}(Y)}\left(\lambda, x_{0,1}, \ldots, x_{k, j}, \ldots, x_{l, r(l)}\right) .
\end{gathered}
$$

From Definition 2.7 it is immediate that

$$
\begin{aligned}
F_{1}\left(\lambda^{m_{1}} \mu_{1}, \ldots, \lambda^{m_{m}} \mu_{m}\right) & \in \mathbb{Q}\left(\lambda^{\frac{1}{2}}, \mu_{1}^{\frac{1}{2}}, \ldots, \mu_{m}^{\frac{1}{2}}\right), \\
F_{2, V}\left(\lambda^{m_{1}} \mu_{1}, \ldots, \lambda^{m_{m}} \mu_{m}\right) & \in \mathbb{Q}\left[\lambda^{ \pm \frac{1}{2}}, \mu_{1}^{ \pm \frac{1}{2}}, \ldots, \mu_{m}^{ \pm \frac{1}{2}}\right][[q]]
\end{aligned}
$$

and

$$
\begin{aligned}
G_{1, \mathcal{N}}\left(\lambda, x_{1}, \ldots, x_{m}\right) & \in \prod_{m_{i}=0} \frac{1}{x_{i}} \cdot \mathbb{Q}\left[\frac{1}{\lambda^{m_{i}}-1} \mid m_{i} \neq 0\right]\left[\lambda^{ \pm \frac{1}{2}}\right]\left[\left[x_{1}, \ldots, x_{m}\right]\right], \\
G_{2, V, \mathcal{N}}\left(\lambda, x_{1}, \ldots, x_{m}\right) & \in \mathbb{Q}\left[\lambda^{ \pm \frac{1}{2}}\right]\left[\left[x_{1}, \ldots, x_{m}\right]\right][[q]] .
\end{aligned}
$$

Lemma 2.12. The expression $G_{V, \mathcal{N}(Y)}$ is in the image of $\iota$.

Proof. It follows directly from the definitions that $G_{V, \mathcal{N}(Y)}$ is an element in $\mathcal{A}\left[\left[x_{k, j} \mid\right.\right.$ $(k, j) \in C(Y)]]$ where $\mathcal{A}$ was defined as $\mathcal{A}=\mathbb{Q}\left(\lambda^{\frac{1}{2}}\right)[[q]]$. By Lemma 2.10 the image of $\iota$ is equal to the invariants of the action $W$ on $\mathcal{A}\left[\left[x_{k, j} \mid(k, j) \in C(Y)\right]\right]$. This action $W$ is given by arbitrary permutations of the $x_{k, j}$ for fixed $k$, and by even numbers of sign changes on the $x_{0, j}$. Since $F_{1}$ is symmetric in all its arguments and an odd function in $x_{i}$ and the rotation numbers $m_{k, j}$ do not depend on $j$ for fixed $k, G_{1, \mathcal{N}(Y)}$ is invariant under $W$. Obviously $\prod_{j=1}^{r(0)} x_{0, j}$ is symmetric in $x_{0, j}$ and an odd function, so again it is invariant under $W$. Since $V$ is a power series of representations of $\operatorname{Spin}(2 \mathrm{~m})$, the formal Chern character $F_{2, V}$ is symmetric in all its arguments and invariant under an even number of sign changes on the $x_{i}$. By definition

$$
\begin{aligned}
G_{2, V, \mathcal{N}(Y)}\left(\lambda, x_{0,1}, \ldots, x_{k, j}, \ldots, x_{l, r(l)}\right) & \\
= & F_{2, V}\left(e^{x_{0,1}}, \ldots, e^{x_{0, r(0)}}, \lambda^{m_{Y}^{1}} \cdot e^{x_{1,1}} \ldots, \lambda^{m_{Y}^{1}} \cdot e^{x_{1, r(1)}}, \ldots,\right. \\
& \left.\lambda^{m_{Y}^{l}} \cdot e^{x_{l, 0}}, \ldots, \lambda^{m_{Y}^{l}} \cdot e^{x_{l, r(l)}}\right) .
\end{aligned}
$$

Hence, $G_{2, V, \mathcal{N}(Y)}$ is invariant under $W$. Therefore $G_{V, \mathcal{N}(Y)}$ is in the image of $\iota$.

Next we consider the map: ${ }^{4}$

$$
\mathcal{A}\left[\left[c_{k, i}, p_{h}, e \mid(k, i) \in C(Y), k \neq 0, h=1, \ldots, r(0)-1\right]\right] \stackrel{t}{\longrightarrow} H^{*}(Y ; \mathcal{A}),
$$

where $t$ is the graded ring homomorphism defined by mapping $c_{k, i}$ to the $i$-th Chern class of the complex bundle $\nu_{k}$, mapping $p_{h}$ to the $h$-th Pontrjagin class of the tangent bundle of $Y$ and $e$ to the Euler class of $Y$.

We are now in the position to rephrase the Lefschetz fixed point formula (cf. Theorem 2.8) in terms of $\iota, t$ and $G_{V, \mathcal{N}(Y)}$.

\footnotetext{
${ }^{4}$ This is the map in cohomology with coefficients in $\mathcal{A}$ induced by the classifying map between $Y$ and $B G$ (see the previous footnote).
} 
Theorem 2.13 (Lefschetz fixed point formula - Revisited). Let $M$ be a $2 m$ dimensional Spin-manifold with Spin-preserving $S^{1}$-action and let $\mathcal{Y}$ be the set of connected components of $M^{S^{1}}$. Let $V \in R(\operatorname{Spin}(2 m))[[q]]$. Then

$$
\hat{A}_{S^{1}}(M ; V)=\sum_{Y \in \mathcal{Y}} a(Y, V),
$$

where

$$
a(Y, V):=(-1)^{m}\left\langle t \circ \iota^{-1}\left(G_{V, \mathcal{N}(Y)}\left(\lambda, x_{0,1}, \ldots, x_{k, j}, \ldots, x_{l, r(l)}\right)\right),[Y]\right\rangle \in \mathcal{A} .
$$

In the next proposition we establish the main result of this section: We describe the local invariants as linear combinations of coefficients of $G_{V, \mathcal{N}(Y)}$. Recall that $G_{V, \mathcal{N}(Y)}$ is in $\mathcal{A}\left[\left[x_{k, j} \mid(k, j) \in C(Y)\right]\right]$. If we assign to $x_{k, j}$ degree 2 the homogeneous part of $G_{V, \mathcal{N}(Y)}$ of degree $2 r(0)=\operatorname{dim}(Y)$ is an $\mathcal{A}$-linear combination of the monomials $\prod_{(k, j) \in C(Y)} x_{k, j}^{n_{k, j}}$ of degree $2 r(0)$.

Proposition 2.14. Consider the coefficients of the monomials $\prod_{(k, j) \in C(Y)} x_{k, j}^{n_{k, j}}$ of degree $2 r(0)$ in $G_{V, \mathcal{N}(Y)}$ satisfying $n_{k, j} \geq n_{k, j+1}$. The local invariants $a(Y, V)$ are unique $\mathbb{Q}$-linear combinations of these coefficients. The rational coefficients of these linear combinations only depend on the fixed point data $C(Y)$ and the mixed characteristic numbers of $Y$ and the bundles $\nu_{k}$; in particular they do not depend on the chosen power series of representations $V$.

Proof. We use the formula for $a(Y, V)$ given in the Lefschetz fixed point theorem 2.13. Applying $\langle t(\cdot),[Y]\rangle$ to $\iota^{-1}\left(G_{V, \mathcal{N}(Y)}\right)$ amounts to extracting the degree $2 r(0)$ part and substituting the corresponding mixed characteristic numbers of $Y$ and the bundles $\nu_{k}$ for the monomials in $c_{k, i}, p_{h}$, and $e$. The result is an integer linear combination of the coefficients of $\iota^{-1}\left(G_{V, \mathcal{N}(Y)}\right)$ with respect to the monomials in $c_{k, i}, p_{h}$, and $e$. By Lemma 2.10 these coefficients are unique $\mathbb{Q}$-linear combinations of the coefficients of the monomials $\prod_{(k, j) \in C(Y)} x_{k, j}^{n_{k, j}}$ in $G_{V, \mathcal{N}(Y)}$ of the same degree (with $n_{k, j} \geq n_{k, j+1}$ ), which do not depend on $G_{V, \mathcal{N}(Y)}$.

2.6. Exponential Representations and Differential Operators. In this section we will simplify the main result of the previous section in the case of exponential representations. For any exponential representation $V$ we will define the building block $f_{V}$. The equivariant index of the twisted Dirac operator will then be expressed as the result of applying a certain operator polynomial to $f_{V}$. The operator polynomial only depends on the $S^{1}$-action over the fixed point components and not on the chosen exponential representation $V$. This result leads to the definition of DSC-rings. We will see that if the building block associated to the exponential representation lies in some DSC-ring, then the equivariant index lies in the same ring. We will make use of this statement as a tool to derive properties of the equivariant index from properties of the building block in Chapter 3.

Definition 2.15. $\quad$ 1. A power series of representations $V \in R(\operatorname{Spin}(2 m))[[q]]$ is called an exponential representation iff its formal Chern character $F_{2, V} \in \mathbb{Q}\left[\mu_{1}^{ \pm \frac{1}{2}}, \ldots, \mu_{m}^{ \pm \frac{1}{2}}\right][[q]]$ is of the form $F_{2, V}=\prod_{i=1}^{m} f_{2, V}\left(\mu_{i}\right)$, where $f_{2, V} \in$ $\mathbb{Q}\left[\mu^{ \pm \frac{1}{2}}\right][[q]]$ 
2. For an exponential representation $V$ with $F_{2, V}=\prod_{i=1}^{m} f_{2, V}\left(\mu_{i}\right)$ we define the building block

$$
f_{V}(\mu):=\frac{1}{\mu^{\frac{1}{2}}-\mu^{-\frac{1}{2}}} \cdot f_{2, V}(\mu) \in \frac{1}{\mu^{\frac{1}{2}}-\mu^{-\frac{1}{2}}} \cdot \mathbb{Q}\left[\mu^{ \pm \frac{1}{2}}\right][[q]] .
$$

As an example the power series of representations $R$ and $R^{0}$ defining elliptic genera are exponential with the building blocks

$$
f_{R}(\mu)=\frac{\left(\mu^{\frac{1}{2}}+\mu^{-\frac{1}{2}}\right)}{\left(\mu^{\frac{1}{2}}-\mu^{-\frac{1}{2}}\right)} \prod_{n=1}^{\infty} \frac{\left(1+q^{n} \mu\right)\left(1+q^{n} \mu^{-1}\right)\left(1-q^{n}\right)^{2}}{\left(1-q^{n} \mu\right)\left(1-q^{n} \mu^{-1}\right)\left(1+q^{n}\right)^{2}}
$$

and

$$
f_{R^{0}}(\mu)=\frac{1}{\left(\mu^{\frac{1}{2}}-\mu^{-\frac{1}{2}}\right)} \prod_{n=1}^{\infty} \frac{\left(1-q^{2 n-1} \mu\right)\left(1-q^{2 n-1} \mu^{-1}\right)\left(1-q^{2 n}\right)^{2}}{\left(1-q^{2 n} \mu\right)\left(1-q^{2 n} \mu^{-1}\right)\left(1-q^{2 n-1}\right)^{2}} .
$$

Note that $x \cdot f_{R}\left(e^{x}\right)$ (resp. $\left.x \cdot f_{R^{0}}\left(e^{x}\right)\right)$ is the characteristic power series of the elliptic genus $\varphi$ (resp. $\varphi^{0}$ ) in the Hirzebruch formalism.

For an exponential representation $V$ the expression $G_{V, \mathcal{N}(Y)}$ simplifies in the following way:

$$
G_{V, \mathcal{N}(Y)}\left(\lambda, x_{0,1}, \ldots, x_{k, j}, \ldots, x_{l, r(l)}\right)=\prod_{j=1}^{r(0)} x_{0, j} \cdot \prod_{k=0}^{l} \prod_{j=1}^{r(k)} f_{V}\left(\lambda^{m_{Y}^{k}} e^{x_{k, j}}\right) .
$$

Definition 2.16. For $r \neq 0$ and $i \geq 0$ let $\partial_{i}(r): \mathbb{Q}\left(\lambda^{\frac{1}{2}}\right)[[q]] \longrightarrow \mathbb{Q}\left(\lambda^{\frac{1}{2}}\right)[[q]]$ be the operator defined by

$$
\partial_{i}(r)(f):=\frac{1}{i ! \cdot r^{i}} \cdot \frac{\partial^{i}}{\partial z^{i}}\left(f\left(\lambda^{r}\right)\right), \quad \text { where } \lambda=e^{z} .
$$

For $f \in \mathbb{Q}\left(\lambda^{\frac{1}{2}}\right)[[q]]$ such that $x f\left(e^{x}\right) \in \mathbb{Q}[[x]][[q]]$ we define

$$
\partial_{i}(0)(f):=\frac{1}{(i+1) !} \cdot \frac{\partial^{i+1}}{\partial x^{i+1}}\left(x f\left(e^{x}\right)\right)(0) \quad \text { for } i \geq-1 .
$$

We give the operator $\partial_{i}(r)$ degree $i$ for any $r$.

In the next proposition we describe the equivariant index $\hat{A}_{S^{1}}(M ; V)$ as the result of applying a polynomial in the $\partial_{i}(r)$ to the building block.

Proposition 2.17. Let $M$ be a $2 m$-dimensional Spin-manifold with Spin-preserving $S^{1}$-action and let $Y$ be a connected component of $M^{S^{1}}$.

1. There exists a polynomial of degree zero in the operators $\partial_{i}(r)$ with rational coefficients such that for any exponential representation $V \in R(\operatorname{Spin}(2 m))$ $[[q]]$ the local invariants $a(Y, V)$ defined in the Lefschetz fixed point theorem 2.13 are the result of applying this operator polynomial to the building block $f_{V}$. More precisely this operator polynomial is a rational linear combination of the monomials $\prod_{(k, j) \in C(Y)} \partial_{n_{k, j}}\left(m_{Y}^{k}\right)$ with $n_{k, j} \geq n_{k, j+1}$ and having degree 0. Especially all monomials have length $m$.

2. There exists a polynomial $\mathfrak{P}_{M}$ of degree zero in the operators $\partial_{i}(r)$ with rational coefficients such that for any exponential representation $V \in$ $R(\operatorname{Spin}(2 m))[[q]]$ the equivariant index $\hat{A}_{S^{1}}(M ; V)$ is the result of applying this operator polynomial to the building block $f_{V}$, i.e. $\hat{A}_{S^{1}}(M ; V)=\mathfrak{P}_{M}\left(f_{V}\right)$. All monomials in this polynomial have length $\mathrm{m}$. 
Proof. Recall that for any exponential representation $V$ we introduced in Definition 2.15 the building block $f_{V}(\mu) \in \frac{1}{\mu^{\frac{1}{2}}-\mu^{-\frac{1}{2}}} \cdot \mathbb{Q}\left[\mu^{ \pm \frac{1}{2}}\right][[q]]$. Note that $f_{V}\left(\lambda^{r} e^{x}\right) \in$ $\mathbb{Q}\left(\lambda^{\frac{1}{2}}\right)[[x]][[q]]$ for $r \neq 0$ and $f_{V}\left(\lambda^{r} e^{x}\right) \in \frac{1}{x} \mathbb{Q}[[x]][[q]]$ for $r=0$. Let $a_{V, i}(r) \in$ $\mathbb{Q}\left(\lambda^{\frac{1}{2}}\right)[[q]]$ be the coefficient of $x^{i}$ in $f_{V}\left(\lambda^{r} e^{x}\right)$. So for $r \neq 0$

where $\lambda=e^{z}$, and

$$
\begin{aligned}
a_{V, i}(r) & =\frac{1}{i !} \cdot \frac{\partial^{i}}{\partial x^{i}}\left(f_{V}\left(\lambda^{r} e^{x}\right)\right)(0) \\
& =\frac{1}{i ! \cdot r^{i}} \cdot \frac{\partial^{i}}{\partial z^{i}}\left(f_{V}\left(\lambda^{r}\right)\right) \in \mathbb{Q}\left(\lambda^{\frac{1}{2}}\right)[[q]],
\end{aligned}
$$

$$
a_{V, i}(0)=\frac{1}{(i+1) !} \cdot \frac{\partial^{i+1}}{\partial x^{i+1}}\left(x f_{V}\left(e^{x}\right)\right)(0) \in \mathbb{Q}[[q]] .
$$

From the definition of $\partial_{i}(r)$ it is obvious that $a_{V, i}(r)=\partial_{i}(r)\left(f_{V}\right)$. So Part 1 of the proposition follows directly from Proposition 2.14 and formula (7). For Part 2 recall that $\hat{A}_{S^{1}}(M ; V)=\sum_{Y \in \mathcal{Y}} a(Y, V)$.

We will now study this result from a more abstract point of view. For this we define the following operators on the ring $\mathcal{T}:=\mathbb{C}\left[\frac{1}{z}\right][[z]][[q]]$ :

$$
\begin{aligned}
& \partial_{i}: \mathcal{T} \rightarrow \mathcal{T}, \partial_{i}(f):=\frac{\partial^{i}}{\partial z^{i}}(f) \quad \text { for } i \in \mathbb{N}, \\
& \iota_{r}: \mathcal{T} \rightarrow \mathcal{T}, \iota_{r}(f)(z):=f(r z) \quad \text { for } r \in \mathbb{Z} \backslash\{0\} .
\end{aligned}
$$

Let us call $f \in \mathcal{T}$ almost regular if $f \in \mathcal{T}_{0}:=\frac{1}{z} \mathbb{C}[[z]][[q]]$. We define operators $\partial_{i}^{0}$ on $\mathcal{T}_{0}$ by

$$
\partial_{i}^{0}: \mathcal{T}_{0} \rightarrow \mathcal{T}_{0}, \partial_{i}^{0}(f)=\frac{\partial^{i+1}}{\partial z^{i+1}}(z \cdot f)(0) \quad \text { for } i \in \mathbb{Z}, i \geq-1 .
$$

Definition 2.18. A $\mathbb{Z}$-graded $\mathbb{Q}$-algebra $S^{*}$, which is an ungraded subalgebra of $\mathcal{T}$, is called a DSC-ring of series of type $s, s \in \mathbb{Z}$, iff

1. the operation of $\partial_{i}$ and $\iota_{r}$ for $i \in \mathbb{N}$ and $r \in \mathbb{Z} \backslash\{0\}$ restrict to $S^{*}$,

2. the operation of $\partial_{i}^{0}$ for $i \in \mathbb{Z}$ and $i \geq-1$ restricts to the almost regular elements $S_{0}^{*}:=S^{*} \cap \mathcal{T}_{0}$,

3. the operations $\partial_{i}, \iota_{r}$, and $\partial_{i}^{0}$ have degrees $s \cdot i, 0$ and $s \cdot i$, respectively (i.e. they shift degrees by $s \cdot i, 0$ and $s \cdot i$ ).

These rings are called DSC-rings since they are closed under differentiation and scalar multiplication by integers. We will be interested in the case of DSC-rings of series of type 0 or 1 . Observe that any ungraded ring $S$ can be viewed as a graded $\operatorname{ring} S^{*}$ defined by $S^{*}:=S^{0}:=S$. Examples for DSC-rings of series of type 0 which come from ungraded rings in this way are the ring $\mathcal{T}$ itself and the ring $\mathbb{Q}\left(\lambda^{\frac{1}{2}}\right)[[q]]$ via the inclusion to $\mathcal{T}$ given by $\lambda^{\frac{1}{2}} \mapsto e^{\frac{z}{2}}$. Note that for any exponential representation $V$ the building block $f_{V}(\lambda)$ is an almost regular element in the DSC-ring of series $\mathbb{Q}\left(\lambda^{\frac{1}{2}}\right)[[q]]$. The next lemma is trivial.

Lemma 2.19. The action of $\partial_{i}(r):=\frac{1}{i ! r^{i}} \cdot \partial_{i} \circ \iota_{r}$ for $i \in \mathbb{N}$ and $r \in \mathbb{Z} \backslash\{0\}$, respectively of $\partial_{i}(0):=\frac{1}{(i+1) !} \cdot \partial_{i}^{0}$ for $i \in \mathbb{Z}, i \geq-1$, is defined on any DSC-ring of series, respectively its almost regular elements. On the ring $\mathbb{Q}\left(\lambda^{\frac{1}{2}}\right)[[q]]$ it coincides with the action introduced in Definition 2.16. 
The last result of this chapter will become important in the next chapter.

Proposition 2.20. Let $M$ be a $2 m$-dimensional Spin-manifold with Spin-preserving $S^{1}$-action. Let $V$ be an exponential representation of $\operatorname{Spin}(2 m)$, let $S^{*}$ be a $D S C$-ring of series of type $s$ and assume $f_{V}\left(e^{z}\right) \in S^{k}$ for some $k$. Then the equivariant index $\hat{A}_{S^{1}}(M ; V)(\lambda)$ is in $S^{m k}$ for $\lambda=e^{z}$. More precisely, there exists a polynomial $\mathfrak{P}_{M}$ in the operators $\partial_{i}(r)$ such that $\hat{A}_{S^{1}}(M ; V)=\mathfrak{P}_{M}\left(f_{V}\right)$. The polynomial is independent of the chosen exponential representation $V$ and the chosen $D S C$-ring $S^{*}$.

Proof. By Proposition 2.17, Part 2, the equivariant index $\hat{A}_{S^{1}}(M ; V)$ is the result of applying a polynomial $\mathfrak{P}_{M}$ of degree zero in the operators $\partial_{i}(r)$ to the building block $f_{V}$, which is always almost regular. Furthermore each monomial in this polynomial has length $m$. Since by Lemma 2.19 the operators $\partial_{i}(r)$ act on $S^{*}\left(\partial_{i}(r)\right.$ acts on $S_{0}^{*}$ for $r=0$ ), and shift degree by $s \cdot i$, the result follows.

\section{Function-Theoretical Considerations}

3.1. DSC-Rings of Functions. In this section we will prepare the setting which will be used to study elliptic genera as meromorphic functions. We will define a DSC-ring of series and a DSC-ring of meromorphic functions especially adapted to elliptic genera. These two rings will be related by a convergence map $\kappa$.

Let $B \subset \mathbb{C}$ be the open unit disk around 0 and let $\widetilde{\mathbb{C}}^{*}$ be the connected double cover of $\mathbb{C}^{*}$. We denote by $\lambda$, resp. $\lambda^{\frac{1}{2}}$, the coordinate functions on $\mathbb{C}^{*}$, resp. $\widetilde{\mathbb{C}}^{*}$, and define a family of open sets by $B_{N}:=\left\{(q, \lambda) \in B \times\left.\widetilde{\mathbb{C}}^{*}|| q\right|^{\frac{1}{N}}<|\lambda|<|q|^{-\frac{1}{N}}\right\}$ for $N \in \mathbb{N}$.

Definition 3.1. Let $\mathcal{R} \subset \mathbb{C}\left(\lambda^{\frac{1}{2}}\right)[[q]]$ be the set of all elements $f(\lambda)=\sum_{n=0}^{\infty} f_{n}(\lambda) q^{n}$, $f_{n}(\lambda) \in \mathbb{C}\left(\lambda^{\frac{1}{2}}\right)$, for which there exists $a(\lambda) \in \mathbb{C}[\lambda] \backslash\{0\}$, such that $a(\lambda) f_{n}(\lambda)$ is holomorphic on $\widetilde{\mathbb{C}}^{*}$ for all $n$ and $\sum_{n=0}^{\infty} a(\lambda) f_{n}(\lambda) q^{n}$ converges normally on $B_{N}$ for some $N \in \mathbb{N}$.

It is an easy exercise to show that $\mathcal{R}$ is a $\mathbb{Q}$-algebra. We make $\mathcal{R}$ into a graded $\mathbb{Q}$-algebra via $\mathcal{R}^{*}:=\mathcal{R}^{0}:=\mathcal{R}$. The $\mathbb{Q}$-algebra $\mathcal{R}$ can be embedded as a subalgebra of $\mathcal{T}=\mathbb{C}\left[\frac{1}{z}\right][[z]][[q]]$ via $\lambda^{\frac{1}{2}} \mapsto e^{\frac{z}{2}}$.

Lemma 3.2. The ring $\mathcal{R}^{*}$ is a DSC-ring of series of type 0 .

Proof. The condition that the action of $\iota_{r}$ restricts to $\mathcal{R}$ is obviously fulfilled. For the action of $\partial_{i}$ note that if $\sum_{n=0}^{\infty} a(\lambda) f_{n}(\lambda) q^{n}$ converges normally, then also $\sum_{n=0}^{\infty} a^{2}(\lambda) \frac{\partial}{\partial z} f_{n}(\lambda) q^{n}$ converges normally. To show that $\partial_{i}^{0} f=\sum_{n=0}^{\infty} \frac{\partial^{i+1}}{\partial z^{i+1}}\left(z f_{n}\left(e^{z}\right)\right)(0) q^{n}$ converges normally for every almost regular $f$ on some $B_{N}$ we remark that if $a(\lambda)=(\lambda-1)^{k} b(\lambda)$ with $b(\lambda) \in \mathbb{C}[\lambda]$ and $b(1) \neq 0$, then $\frac{\partial^{i+1}}{\partial z^{i+1}}\left(z f_{n}\left(e^{z}\right)\right)(0)$ is a $\mathbb{C}$-linear combination of $\frac{\partial^{j}}{\partial \lambda^{j}}\left(a(\lambda) f_{n}(\lambda)\right)(1)$ for $j \in\{0, \ldots, k+i\}$. From the normal convergence of $\sum_{n=0}^{\infty} a(\lambda) f_{n}(\lambda) q^{n}$ on some $B_{N}$ it follows that $\sum_{n=0}^{\infty} \frac{\partial^{j}}{\partial \lambda^{j}}\left(a(\lambda) f_{n}(\lambda)\right) q^{n}$ also converges normally on $B_{N}$ for all $j$. Since $B \times\{1\} \subset B_{N}$ for all $N$ we get that $\partial_{i}^{0} f$ converges normally on $B_{N}$, so $\partial_{i}^{0} f \in \mathcal{R}$. 
A function on an open subset of $\mathbb{C}^{r}$ is meromorphic iff it is locally the quotient $\frac{f}{g}$ of holomorphic functions $f$ and $g$, where $g \neq 0$. A function on a subset $V$ of $\mathbb{C}^{r}$ is meromorphic iff it extends to a meromorphic function on some open neighborhood of $V$. Let $\mathcal{M}(V)$ denote the ring of meromorphic functions on $V$.

For any subset $V=V_{1} \times V_{2}, V_{1} \subset \mathbb{C}^{l}, V_{2} \subset \mathbb{C}$ with $r \cdot V_{2} \subset V_{2}$, for all $r \in \mathbb{Z}$, we define operators $\partial_{i}$ and $\iota_{r}$ on $\mathcal{M}(V)$ by

$$
\begin{aligned}
& \partial_{i}: \mathcal{M}(V) \rightarrow \mathcal{M}(V), \partial_{i}(f)(\xi, z):=\frac{\partial^{i}}{\partial z^{i}}(f)(\xi, z) \quad \text { for } i \in \mathbb{N}, \\
& \iota_{r}: \mathcal{M}(V) \rightarrow \mathcal{M}(V), \iota_{r}(f)(\xi, z):=f(\xi, r \cdot z) \quad \text { for } r \in \mathbb{Z} \backslash\{0\} .
\end{aligned}
$$

Let us call $f \in \mathcal{M}(V)$ almost regular if $f$ is an element in

$$
\mathcal{M}(V)_{0}:=\left\{g \in \mathcal{M}(V) \mid z \cdot g \text { is holomorphic on } V_{1} \times\{0\} \subset V\right\} .
$$

We define operators $\partial_{i}^{0}$ on $\mathcal{M}(V)_{0}$ by

$$
\partial_{i}^{0}: \mathcal{M}(V)_{0} \rightarrow \mathcal{M}(V)_{0}, \partial_{i}^{0}(f)(\xi, z)=\frac{\partial^{i+1}}{\partial z^{i+1}}(z \cdot f)(\xi, z)(z=0),
$$

for $i \in \mathbb{Z}, i \geq-1$. In particular, the operators $\partial_{i}$ and $\iota_{r}$ (resp. $\left.\partial_{i}^{0}\right)$ act on $\mathcal{M}(B \times i \mathbb{R})$ (resp. $\left.\mathcal{M}(B \times i \mathbb{R})_{0}\right)$. The ring $\mathcal{M}\left(B \times S^{1}\right)$ may be embedded into $\mathcal{M}(B \times i \mathbb{R})$ via $i \mathbb{R} \rightarrow S^{1}, z \mapsto e^{z}=\lambda$, and the action of $\partial_{i}$ and $\iota_{r}$ restricts to $\mathcal{M}\left(B \times S^{1}\right)$. The action on $\mathcal{M}\left(B \times S^{1}\right)$ will also be denoted by $\partial_{i}$, and $\iota_{r}$ (here we abuse notation; the operators are distinguished by the variables $z$ and $\lambda$ ). It is given by

$$
\partial_{i}: \mathcal{M}\left(B \times S^{1}\right) \rightarrow \mathcal{M}\left(B \times S^{1}\right), \partial_{i}(f)(q, \lambda):=\frac{\partial^{i}}{\partial z^{i}}(f)(q, \lambda) \quad \text { for } i \in \mathbb{N},
$$

where $\lambda=e^{z}$,

$$
\iota_{r}: \mathcal{M}\left(B \times S^{1}\right) \rightarrow \mathcal{M}\left(B \times S^{1}\right), \iota_{r}(f)(q, \lambda):=f\left(q, \lambda^{r}\right) \quad \text { for } r \in \mathbb{Z} \backslash\{0\} .
$$

Let us call $f \in \mathcal{M}\left(B \times S^{1}\right)$ almost regular if $f$ is an element in

$$
\begin{aligned}
\mathcal{M}\left(B \times S^{1}\right)_{0}:=\left\{g \in \mathcal{M}\left(B \times S^{1}\right) \mid\right. & \\
& \left.(\lambda-1) \cdot g \text { is holomorphic on } B \times\{1\} \subset B \times \widetilde{\mathbb{C}}^{*}\right\},
\end{aligned}
$$

so $f$ is almost regular iff its image in $\mathcal{M}(B \times i \mathbb{R})$ is almost regular. Again, the action of $\partial_{i}^{0}$ on $\mathcal{M}(B \times i \mathbb{R})_{0}$ restricts to $\mathcal{M}\left(B \times S^{1}\right)_{0}$ and is given by

$$
\partial_{i}^{0}: \mathcal{M}\left(B \times S^{1}\right)_{0} \rightarrow \mathcal{M}\left(B \times S^{1}\right)_{0}, \partial_{i}^{0}(f)(q, \lambda)=\frac{\partial^{i+1}}{\partial z^{i+1}}(z \cdot f)(q, \lambda)(z=0),
$$

for $i \in \mathbb{Z}, i \geq-1$, where $\lambda=e^{z}$.

Definition 3.3. A $\mathbb{Z}$-graded $\mathbb{Q}$-algebra $S^{*}$, which is an ungraded subalgebra of $\mathcal{M}\left(B \times S^{1}\right)$, is called a DSC-ring of functions of type $s, s \in \mathbb{Z}$, iff

1. the operation of $\partial_{i}$ and $\iota_{r}$ for $i \in \mathbb{N}$ and $r \in \mathbb{Z} \backslash\{0\}$ restrict to $S^{*}$,

2. the operation of $\partial_{i}^{0}$ for $i \in \mathbb{Z}$ and $i \geq-1$ restricts to the almost regular elements $S_{0}^{*}:=S^{*} \cap \mathcal{M}\left(B \times S^{1}\right)_{0}$,

3. the operations $\partial_{i}, \iota_{r}$, and $\partial_{i}^{0}$ have degrees $s \cdot i, 0$ and $s \cdot i$, respectively (i.e. they shift degrees by $s \cdot i, 0$ and $s \cdot i$ ).

The ring $\mathcal{M}\left(B \times S^{1}\right)$ itself is a DSC-ring of functions of type 0 , which is concentrated in degree 0 . The next lemma is trivial. 
Lemma 3.4. The action of $\partial_{i}(r):=\frac{1}{i ! r^{i}} \cdot \partial_{i} \circ \iota_{r}$ for $i \in \mathbb{N}$ and $r \in \mathbb{Z} \backslash\{0\}$, respectively of $\partial_{i}(0):=\frac{1}{(i+1) !} \cdot \partial_{i}^{0}$ for $i \in \mathbb{Z}, i \geq-1$, is defined on any DSC-ring of functions, respectively its almost regular elements. If the ring is of type $s$, they shift degree by $i \cdot s$.

Definition 3.5. A homomorphism of DSC-rings (of series or functions) $\gamma$ : $S_{0}^{*} \rightarrow S_{1}^{*}$ is a $\mathbb{Q}$-algebra homomorphism which maps almost regular elements to almost regular elements and commutes with the action of $\partial_{i}, \iota_{r}$ and $\partial_{i}^{0}$. The homomorphism does not have to be graded.

From the definition of a homomorphism the following property is obvious. Recall the definition of $\mathfrak{P}_{M}$ as a polynomial in the operators $\partial_{i}(r)$ from Proposition 2.17, Part 2.

Lemma 3.6. Any homomorphism $\gamma: S_{0}^{*} \rightarrow S_{1}^{*}$ of DSC-rings commutes with the action of the operators $\partial_{i}(r)$ for $i \geq 0, r \in \mathbb{Z}$. Especially, if $f \in\left(S_{0}^{*}\right)_{0}$ (i.e. $f$ is almost regular), then $\gamma\left(\mathfrak{P}_{M}(f)\right)=\mathfrak{P}_{M}(\gamma(f))$. Furthermore for $f$ of degree $k$, $\mathfrak{P}_{M}(f)$ has degree $m k$.

Definition 3.7. We define the limit map $\kappa: \mathcal{R} \rightarrow \mathcal{M}\left(B \times S^{1}\right)$ in the following way: If $f=\sum_{n=0}^{\infty} f_{n}(\lambda) q^{n} \in \mathcal{R}$ and $a(\lambda) \in \mathbb{C}[\lambda] \backslash\{0\}$ s.t. $a(\lambda) f_{n}(\lambda)$ is holomorphic on $\widetilde{\mathbb{C}}^{*}$ and $\sum_{n=0}^{\infty} a(\lambda) f_{n}(\lambda) q^{n}$ converges normally to a function $\tilde{f}(\lambda, q)$ on some $B_{N}$, then let $\kappa(f):=\frac{\tilde{f}(\lambda, q)}{a(\lambda)}$.

Lemma 3.8. The limit map $\kappa$ is a well-defined injective homomorphism of $\mathbb{Q}$ algebras.

Proof. By an easy argument the map $\kappa$ does not depend on the chosen function $a(\lambda)$. So $\kappa$ is well-defined and it is obviously also a homomorphism of $\mathbb{Q}$-algebras. For the injectivity of $\kappa$ note that if $\kappa(f)=0$, then $\sum_{n=0}^{\infty} a(\lambda) f_{n}(\lambda) q^{n}$ converges normally to the 0 -function. By taking derivatives with respect to $q$ all $a(\lambda) f_{n}(\lambda)$ vanish identically, so all $f_{n}$ vanish.

Lemma 3.9. Let $f \in \mathcal{R}$, let $a(\lambda) \in \mathbb{C}[\lambda] \backslash\{0\}$ and let $\tilde{f}$ be a holomorphic function s.t. $\kappa(f)=\frac{\tilde{f}(\lambda, q)}{a(\lambda)}$ on some $B_{N}$. Let $\mathcal{Z}(a)=\left\{\lambda \in \widetilde{\mathbb{C}}^{*} \mid a(\lambda)=0\right\}$ be the finite set of zeroes of a on $\widetilde{\mathbb{C}}^{*}$. Then $\sum_{n=0}^{\infty} f_{n}(\lambda) q^{n}$ converges normally on $B_{N} \backslash(B \times \mathcal{Z}(a))$ to $\kappa(f)$. So $\kappa(f)$ is a meromorphic extension of the holomorphic function $\sum_{n=0}^{\infty} f_{n}(\lambda) q^{n}$ from $B_{N} \backslash(B \times \mathcal{Z}(a))$ to $B_{N}$.

Proof. The lemma is a direct consequence of the definition of normal convergence, since the norm of $a(\lambda)$ for $\lambda$ in any compact subset of $\widetilde{\mathbb{C}}^{*} \backslash \mathcal{Z}(a)$ is greater than 0 .

3.2. Holomorphicity on $S^{1}$. In this section we will show that the elliptic genera $\varphi_{S^{1}}(M)$ and $\varphi_{S^{1}}^{0}(M)$ converge to holomorphic functions on a neighborhood of $B \times S^{1}$ in $B \times \widetilde{\mathbb{C}}^{*}$. To show holomorphicity we will relate these functions to the functions coming from the building blocks by showing that the convergence map $\kappa$ is a homomorphism of DSC-rings. 
Proposition 3.10. Let $U$ be a domain in $\mathbb{C} \times \mathbb{C}$. Consider a series $b=\sum_{n=0}^{\infty} b_{n}$ of holomorphic functions $b_{n}$ on $U$ such that $b$ converges normally on $U^{\prime}=\{(q, \lambda) \in$ $\left.U \mid \lambda \neq \lambda_{0}\right\}$ for some $\lambda_{0} \in \mathbb{C}$. Then $b$ converges normally on all of $U$.

Proof. To prove normal convergence it suffices to show that any point in $U$ lies in the interior of a compact subset of $U$, on which the series converges absolutely in the maximum norm. Since $b$ converges normally on $U^{\prime}$, we only have to check normal convergence on some compact neighborhood of any point of the form $\left(q_{0}, \lambda_{0}\right) \in U$. We take as a compact neighborhood the product $K=D_{q} \times D_{\lambda}$ of two small closed disks around $q_{0}$ and $\lambda_{0}$ in $U$. Since $K$ is compact any function $b_{n}$ takes its maximum on $K$ in some point $\left(q^{\prime}, \lambda^{\prime}\right)$. Since $b_{n}$ is holomorphic, it is also holomorphic in $\lambda$ for fixed $q$. So by the maximum principle we can assume that $\lambda^{\prime}$ lies on the boundary of the disk $D_{\lambda}$, i.e.

$$
\left|b_{n}\right|_{K}=\left|b_{n}\right|_{D_{q} \times \partial D_{\lambda}} .
$$

Since $D_{q} \times \partial D_{\lambda}$ is a compact subset of $U^{\prime}$, the normal convergence of $b=\sum_{n=0}^{\infty} b_{n}$ on $U$ now follows from the normal convergence of the series on $U^{\prime}$.

From this proposition we get the following corollary about the DSC-ring $\mathcal{R}$ of series.

Corollary 3.11. Let $f=\sum_{n=0}^{\infty} f_{n}(\lambda) q^{n} \in \mathcal{R}$ and let $b \in \mathbb{C}[\lambda] \backslash\{0\}$ such that $b(\lambda) f_{n}(\lambda)$ is holomorphic on $\widetilde{\mathbb{C}}^{*}$. Then $\sum_{n=0}^{\infty} b(\lambda) f_{n}(\lambda) q^{n}$ converges normally on $B_{N}$ for some $N>0$.

Proof. Since $f \in \mathcal{R}$, there is a polynomial $a \in \mathbb{C}[\lambda] \backslash\{0\}$ such that $a(\lambda) f_{n}(\lambda)$ is holomorphic on $\widetilde{\mathbb{C}}^{*}$ and $\sum_{n=0}^{\infty} a(\lambda) f_{n}(\lambda) q^{n}$ converges normally on $B_{N}$ for some $N>0$. By Lemma 3.9 we know that $\sum_{n=0}^{\infty} f_{n}(\lambda) q^{n}$ converges normally on $B_{N} \backslash(B \times \mathcal{Z}(a))$, where $\mathcal{Z}(a)=\left\{\lambda \in \widetilde{\mathbb{C}}^{*} \mid a(\lambda)=0\right\}$ is the finite set of zeroes of $a$ on $\widetilde{\mathbb{C}}^{*}$. Thus also $\sum_{n=0}^{\infty} b(\lambda) f_{n}(\lambda) q^{n}$ converges normally on $B_{N} \backslash(B \times \mathcal{Z}(a))$. But since all summands are by assumption actually holomorphic, by Proposition 3.10 this sum converges normally on all of $B_{N}$.

Lemma 3.12. The limit map $\kappa: \mathcal{R} \rightarrow \mathcal{M}\left(B \times S^{1}\right)$ is a homomorphism of DSCrings.

Proof. We already know from Lemma 3.8 that $\kappa$ is a homomorphism of $\mathbb{Q}$-algebras. The fact that $\kappa$ commutes with the action of $\iota_{r}$ is immediate. Now if $\sum_{n=0}^{\infty} a(\lambda) f_{n}(\lambda) q^{n}$ converges to some function $\tilde{f}$, so $\kappa(f)=\frac{\tilde{f}}{a}$, and $\sum_{n=0}^{\infty} a^{2}(\lambda) \frac{\partial}{\partial \lambda} f_{n}(\lambda) q^{n}$ converges to some function $\tilde{g}$, so $\kappa\left(\frac{\partial}{\partial \lambda} f\right)=\frac{\tilde{g}}{a^{2}}$, one can easily check that $\frac{\tilde{g}}{a^{2}}=\frac{\partial}{\partial \lambda} \frac{\tilde{f}}{a}$. So $\kappa$ commutes with $\frac{\partial}{\partial \lambda}$ and by the chain rule also with $\partial_{1}=\frac{\partial}{\partial z}$ for $\lambda=e^{z}$. By induction $\kappa$ commutes with $\partial_{i}$ for all $i>0$. Now we want to show that $\kappa$ maps almost regular elements to almost regular elements. If $f \in \mathcal{R}_{0}$ is any almost regular element, then 
it follows from Corollary 3.11 that one can choose the element $a \in \mathbb{C}[\lambda]$, s.t. $\lambda-1$ divides $a$ only once. Hence $\kappa(f)=\frac{\tilde{f}}{a}$ is obviously almost regular. Finally we have to show that $\kappa$ commutes with $\partial_{i}^{0}$ for all $i \geq-1$. In the proof of Lemma 3.2 we used the fact that if $a(\lambda)=(\lambda-1) b(\lambda)$ with $b(\lambda) \in \mathbb{C}[\lambda]$ and $b(1) \neq 0$, then $\frac{\partial^{i+1}}{\partial z^{i+1}}\left(z f_{n}\left(e^{z}\right)\right)(0)$ is a $\mathbb{C}$-linear combination of $\frac{\partial^{j}}{\partial \lambda^{j}}\left(a(\lambda) f_{n}(\lambda)\right)(1)$ for $j \in\{0, \ldots, i+1\}$. Since the same formula holds for the function $\kappa(f)$ instead of $f$, and $\kappa$ commutes with all $\frac{\partial^{j}}{\partial \lambda^{j}}$ it follows that $\kappa$ also commutes with $\partial_{i}^{0}$ for all $i \geq-1$.

Definition 3.13. Let $V \in R(\operatorname{Spin}(2 m))[[q]]$ be an exponential representation such that the building block $f_{V}(\lambda)$ is an element in $\mathcal{R}$. We define a function $\psi_{V}$ in $\mathcal{M}\left(B \times S^{1}\right)$ by

$$
\psi_{V}:=\kappa\left(f_{V}\right)
$$

Let furthermore $M$ be a $2 m$-dimensional Spin-manifold with Spin-preserving $S^{1}$ action. We define a function $\psi_{M, V}$ in $\mathcal{M}\left(B \times S^{1}\right)$ by

$$
\psi_{M, V}:=\kappa\left(\hat{A}_{S^{1}}(M ; V)\right) .
$$

By Proposition 2.20 the function $\psi_{M, V}$ is well-defined, i.e. the series $\hat{A}_{S^{1}}(M ; V)$ actually lies in $\mathcal{R}$. The next proposition shows that the equivariant index $\hat{A}_{S^{1}}(M ; V)$ converges to a function that is actually holomorphic on $B \times S^{1}$. It also relates this function to the function coming from the building block of $V$.

Proposition 3.14. Let $M$ be a $2 m$-dimensional Spin-manifold with Spin-preserving $S^{1}$-action and let $V \in R(\operatorname{Spin}(2 m))[[q]]$ be an exponential representation such that the building block $f_{V}(\lambda)$ is an element in $\mathcal{R}$. Let $\mathfrak{P}_{M}$ be the operator polynomial defined in Proposition 2.17, Part 2.

1. The functions $\psi_{V}=\kappa\left(f_{V}\right)$ and $\psi_{M, V}=\kappa\left(\hat{A}_{S^{1}}(M ; V)\right)$ are related in the following way:

$$
\psi_{M, V}=\mathfrak{P}_{M}\left(\psi_{V}\right) .
$$

2. The function $\psi_{M, V}$ is holomorphic in some neighborhood $B_{N}$ of $B \times S^{1} \subset$ $B \times \widetilde{\mathbb{C}}^{*}$.

Proof. For the first statement we recall from Definition 3.13 that $\psi_{M, V}$ $=\kappa\left(\hat{A}_{S^{1}}(M ; V)\right)$ and from Proposition 2.20 that $\hat{A}_{S^{1}}(M ; V)=\mathfrak{P}_{M}\left(f_{V}\right)$. Since $\kappa$ is a homomorphism of DSC-rings it commutes with the operator polynomial $\mathfrak{P}_{M}$ (cf. Lemma 3.6) and we get

$$
\psi_{M, V}=\kappa\left(\hat{A}_{S^{1}}(M ; V)\right)=\kappa\left(\mathfrak{P}_{M}\left(f_{V}\right)\right)=\mathfrak{P}_{M}\left(\kappa\left(f_{V}\right)\right)=\mathfrak{P}_{M}\left(\psi_{V}\right) .
$$

The second statement follows immediately from Corollary 3.11: Since we have $\psi_{M, V}=\kappa\left(\hat{A}_{S^{1}}(M ; V)\right)$ and $\hat{A}_{S^{1}}(M ; V)$ as a series of characters of $S^{1}$ actually lies in $\mathbb{Q}\left[\lambda, \lambda^{-1}\right][[q]]$ (i.e. all coefficients are Laurent polynomials), one can choose $b=1$ in Corollary 3.11, so $\kappa\left(\hat{A}_{S^{1}}(M ; V)\right)$ is holomorphic on some $B_{N}$.

Now we want to apply this result to elliptic genera. The next lemma will be used to show that the building blocks defining elliptic genera are in $\mathcal{R}$.

Lemma 3.15. Let $g(\lambda)$ be a holomorphic function on an open subset $U$ of $\mathbb{C}^{r}$. 
1. The series $\sum_{n=0}^{\infty} f_{n} q^{n}=1+g q+g q^{2}+\left(g^{2}+g\right) \cdot q^{3}+\ldots$ defined as a q-power series by the infinite product $\prod_{n=1}^{\infty}\left(1+g q^{n}\right)$ converges normally on $B \times U$.

2. Let $V$ be an open subset of $B \times U$ on which $|g(\lambda) \cdot q|<1$. Then the series $\sum_{n=0}^{\infty} f_{n} q^{n}=1+g q+\left(g^{2}+g\right) \cdot q^{2}+\left(g^{3}+g^{2}+g\right) \cdot q^{3}+\left(g^{4}+g^{3}+2 g^{2}+g\right) \cdot q^{4}+\ldots$ defined as a q-power series by the infinite product $\prod_{n=1}^{\infty} \frac{1}{\left(1-g q^{n}\right)}$ converges normally on $V$.

Proof. We will use the following elementary fact (cf. [Ah66], p. 191): Let $\left\{u_{n}\right\}_{n \in \mathbb{N}}$ be a sequence of complex numbers. Then the product $\Pi_{n=1}^{\infty}\left(1+u_{n}\right)$ converges absolutely iff $\sum_{n=1}^{\infty} u_{n}$ does.

Ad 1: Let $K \subset B \times U$ be a compact set. Without loss of generality we may assume that $K$ has the form $K=K_{1} \times K_{2}$, where $K_{1} \subset B$ and $K_{2} \subset U$. Choose $\left(q_{0}, \lambda_{0}\right) \in K$ for which $\left|g\left(\lambda_{0}\right) \cdot q_{0}\right|$ is equal to $|g(\lambda) \cdot q|_{K}$. Let $\sum_{n=0}^{\infty} a_{n} q^{n}$ be the $q$ power series defined by the infinite product $\prod_{n=1}^{\infty}\left(1+\left|g\left(\lambda_{0}\right)\right| q^{n}\right)$. By the quotient criterion the series $\sum_{n=1}^{\infty}\left|g\left(\lambda_{0}\right) \cdot q_{0}^{n}\right|$ converges to a finite value. Now the above given fact implies that $\sum_{n=0}^{\infty} a_{n}\left|q_{0}\right|^{n}$ also converges to a finite value. Applying the triangle inequality gives

$$
\sum_{n=0}^{\infty}\left|f_{n}(\lambda) \cdot q^{n}\right|_{K} \leq \sum_{n=0}^{\infty} a_{n}\left|q_{0}\right|^{n}<\infty .
$$

Ad 2: Let $K \subset V$ be a compact set. Without loss of generality we may assume that $K$ has the form $K=K_{1} \times K_{2}$, where $K_{1} \subset B$ and $K_{2} \subset U$. Choose $\left(q_{0}, \lambda_{0}\right) \in K$ for which $\left|g\left(\lambda_{0}\right) \cdot q_{0}\right|$ is equal to $|g(\lambda) \cdot q|_{K}$. By assumption $\left|g\left(\lambda_{0}\right) \cdot q_{0}\right|<1$. Let $b_{n}$ be defined as $\frac{\left|g\left(\lambda_{0}\right) \cdot q_{0}^{n}\right|}{\left(1-\left|g\left(\lambda_{0}\right) \cdot q_{0}^{n}\right|\right)}$, thus $1+b_{n}=\frac{1}{\left(1-\left|g\left(\lambda_{0}\right) \cdot q_{0}^{n}\right|\right)}$. Let $\sum_{n=0}^{\infty} a_{n} q^{n}$ be the $q$-power series defined by the infinite product $\prod_{n=1}^{\infty} \frac{1}{\left(1-\left|g\left(\lambda_{0}\right)\right| \cdot q^{n}\right)}=\prod_{n=1}^{\infty}\left(1+b_{n}\right)$. By the quotient criterion the series $\sum_{n=1}^{\infty} b_{n}$ converges to a finite value. Now the above given fact implies that $\sum_{n=0}^{\infty} a_{n}\left|q_{0}\right|^{n}$ also converges to a finite value. By the triangle inequality

$$
\sum_{n=0}^{\infty}\left|f_{n}(\lambda) \cdot q^{n}\right|_{K} \leq \sum_{n=0}^{\infty} a_{n}\left|q_{0}\right|^{n}<\infty
$$

Recall from formulas (5) and (6) of Section 2.6 the building blocks $f_{R}$ and $f_{R^{0}}$ defining elliptic genera.

Proposition 3.16. The q-power series $(\lambda-1) f_{R}(\lambda)$ and $(\lambda-1) f_{R^{0}}(\lambda)$ converge normally on $B_{1}$. In particular, the building blocks $f_{R}(\lambda)$ and $f_{R^{0}}(\lambda)$ of the exponential representations $R$ and $R^{0}$ are elements in $\mathcal{R}$. 
Proof. It follows from Lemma 3.15 and basic properties of normal convergence that the $q$-power series defined by the infinite products

$$
\prod_{n=1}^{\infty} \frac{\left(1+q^{n} \lambda\right)\left(1+q^{n} \lambda^{-1}\right)\left(1-q^{n}\right)^{2}}{\left(1-q^{n} \lambda\right)\left(1-q^{n} \lambda^{-1}\right)\left(1+q^{n}\right)^{2}}
$$

and

$$
\prod_{n=1}^{\infty} \frac{\left(1-q^{2 n-1} \lambda\right)\left(1-q^{2 n-1} \lambda^{-1}\right)\left(1-q^{2 n}\right)^{2}}{\left(1-q^{2 n} \lambda\right)\left(1-q^{2 n} \lambda^{-1}\right)\left(1-q^{2 n-1}\right)^{2}}
$$

converge normally on $B_{1}$. In view of the formulas for $f_{R}$ and $f_{R^{0}}$ (cf. formulas (5) and (6)) the proposition follows directly.

Corollary 3.17. Let $M$ be a $2 m$-dimensional Spin-manifold with Spin-preserving $S^{1}$-action and let $R, R^{0} \in R(\operatorname{Spin}(2 m))[[q]]$ be the exponential representations defining elliptic genera. There exists a positive integer $N(M)$ such that the following holds:

1. The equivariant elliptic genus $\varphi_{S^{1}}(M)=\sum_{n=0}^{\infty} \hat{A}_{S^{1}}\left(M ; R_{n}\right) q^{n}$ at the cusp $i \infty$ converges normally on $B_{N(M)}$ to the holomorphic function $\psi_{M, R}$. In particular, this function has no poles for $\lambda \in S^{1}$.

2. The equivariant elliptic genus $\varphi_{S^{1}}^{0}(M)=\sum_{n=0}^{\infty} \hat{A}_{S^{1}}\left(M ; R_{n}^{0}\right) q^{n}$ at the cusp 0 converges normally on $B_{N(M)}$ to the holomorphic function $\psi_{M, R^{0}}$. In particular, this function has no poles for $\lambda \in S^{1}$.

3. For the functions $\psi_{R}=\kappa\left(f_{R}\right)$ and $\psi_{R^{0}}=\kappa\left(f_{R}^{0}\right)$ there holds

$$
\psi_{M, R}=\mathfrak{P}_{M}\left(\psi_{R}\right) \quad \text { and } \quad \psi_{M, R^{0}}=\mathfrak{P}_{M}\left(\psi_{R^{0}}\right) .
$$

Proof. This is an application of Proposition 3.14 using Proposition 3.16.

3.3. The DSC-Ring of Special Jacobi Functions. In this section we introduce a DSC-ring consisting of functions which transform like Jacobi forms. In the next section we show that equivariant elliptic genera converge to such functions. The modularity properties of these functions with respect to the action of $S L_{2}(\mathbb{Z})$ are then used to finally prove the rigidity theorem.

Let $\mathcal{H}$ be the upper half plane. Now we will recall the action on $\mathcal{M}(\mathcal{H} \times \mathbb{C})$ which is usually used to define Jacobi forms (cf. [EiZa85]).

Let $L$ denote the lattice $2 \mathbb{Z} \times 2 \mathbb{Z}$ and let $\Gamma$ be a subgroup of $S L_{2}(\mathbb{Z})$ of finite index. The group $\Gamma$ acts on the abelian group $L$ by automorphisms, where the action of $A \in \Gamma$ is defined by $(\alpha, \beta) \mapsto(\alpha, \beta) A$. Let $\Gamma \ltimes L$ be the corresponding semi-direct product, i.e. the multiplication in $\Gamma \ltimes L$ is given by

$$
(A,(\alpha, \beta)) \cdot(B,(\gamma, \delta)):=(A \cdot B,(\alpha, \beta) B+(\gamma, \delta)) .
$$

Let $(A, X) \in \Gamma \ltimes L, A=\left(\begin{array}{ll}a & b \\ c & d\end{array}\right), X=(\alpha, \beta)$, and let $f$ be a meromorphic function on $\mathcal{H} \times \mathbb{C}$. For fixed $k \in \mathbb{Z}$ one easily verifies that the assignments

$$
f \mid[A]_{k}(\tau, z):=(c \tau+d)^{-k} \cdot f\left(\frac{a \cdot \tau+b}{c \cdot \tau+d}, \frac{z}{c \cdot \tau+d}\right)
$$

and

$$
f \mid[X](\tau, z):=f(\tau, z+2 \pi i \alpha \tau+2 \pi i \beta)
$$

define an action of $\Gamma \ltimes L$ on the field $\mathcal{M}(\mathcal{H} \times \mathbb{C})$ of meromorphic functions on $\mathcal{H} \times \mathbb{C}$. 
Definition 3.18. We say a meromorphic function $f$ on $\mathcal{H} \times \mathbb{C}$ transforms like a Jacobi form of weight $k$ and index 0 for $\Gamma$ iff

$$
f \mid[A]_{k}(\tau, z)=f(\tau, z) \text { and } f \mid[X](\tau, z)=f(\tau, z)
$$

for any $(A, X) \in \Gamma \ltimes L$.

Definition 3.19. We define the ring $\mathcal{J}_{\Gamma}^{*} \subset \mathcal{M}(\mathcal{H} \times \mathbb{C})$ of special Jacobi functions to be the graded ring of meromorphic functions $f(\tau, z)$ on $\mathcal{H} \times \mathbb{C}$, s.t. $f \in \mathcal{J}_{\Gamma}^{k}$ iff

(i) the function $f$ transforms like a Jacobi form of weight $k$ and index 0 for $\Gamma$,

(ii) the function $f$ has for fixed $\tau$ only poles for $z \in 2 \pi i(\mathbb{Q} \cdot \tau+\mathbb{Q})$,

(iii) for any $A \in S L_{2}(\mathbb{Z})$ there exists a positive integer $N$, s.t. the function $f \mid[A]_{k}$ restricted to $\mathcal{H} \times i \mathbb{R}$ is in the image of the injective map

$$
\begin{aligned}
\mathcal{E}_{N}: \mathcal{M}\left(B \times S^{1}\right) & \rightarrow \mathcal{M}(\mathcal{H} \times i \mathbb{R}), \\
g(q, \lambda) & \mapsto f(\tau, z):=g\left(e^{2 \pi i \tau / N}, e^{z}\right) .
\end{aligned}
$$

Note that in contrast to Jacobi forms these special Jacobi functions are meromorphic with poles on the rational lattice and do not need to fulfill the additional vanishing conditions for the Fourier coefficients (cf. [EiZa85], p. 9).

Remark 3.20. Property (i) implies that $f \mid[A]_{k}$ transforms like a Jacobi form of weight $k$ and index 0 for $A^{-1} \Gamma A$. Since this group has finite index in $S L_{2}(\mathbb{Z})$ there exists an integer $N$ such that $\left(\begin{array}{ll}1 & N \\ 0 & 1\end{array}\right) \in A^{-1} \Gamma A$. Now the matrix $\left(\begin{array}{ll}1 & N \\ 0 & 1\end{array}\right)$ acts by $f(\tau, z) \mapsto f(\tau+N, z)$. So we see that the restriction of $f \mid[A]_{k}$ to $\mathcal{H} \times i \mathbb{R}$ is in the image of $\mathcal{E}_{N}: \mathcal{M}\left(B^{*} \times S^{1}\right) \rightarrow \mathcal{M}(\mathcal{H} \times i \mathbb{R})$, where $N$ depends on $A$ and $\Gamma$ but not on $f$. This means property (iii) only asserts that the meromorphic function $\mathcal{E}_{N}^{-1}\left(f \mid[A]_{k}\right)$ on $B^{*} \times S^{1}$ in fact extends to $B \times S^{1}$.

Since in (iii) the integer $N$ may be chosen to depend only on $A$ and $\Gamma$ (but not on $f$ ) we see for $A=I d$ that the ring $\mathcal{J}_{\Gamma}^{*}$ is a subring of $\mathcal{M}\left(B \times S^{1}\right)$ via $(q, \lambda) \mapsto\left(e^{2 \pi i \tau / N}, e^{z}\right)$. Recall from Section 3.1 that the operators $\iota_{r}$ and $\partial_{i}$ act on $\mathcal{M}(\mathcal{H} \times \mathbb{C})$ and $\mathcal{M}(\mathcal{H} \times i \mathbb{R})$ as well as on $\mathcal{M}\left(B \times S^{1}\right)$. These actions are compatible with the restriction from $\mathcal{M}(\mathcal{H} \times \mathbb{C})$ to $\mathcal{M}(\mathcal{H} \times i \mathbb{R})$ and the embeddings $\mathcal{E}_{N}$ from $\mathcal{M}\left(B \times S^{1}\right)$ to $\mathcal{M}(\mathcal{H} \times i \mathbb{R})$ for all $N$. The same is true for the operators $\partial_{i}^{0}$ acting on the subsets of almost regular functions.

Proposition 3.21. The ring of special Jacobi functions $\mathcal{J}_{\Gamma}^{*}$ is a DSC-ring of functions of type 1 , i.e. for $f \in \mathcal{J}_{\Gamma}^{k}$ holds:

1. $\iota_{r}(f) \in \mathcal{J}_{\Gamma}^{k}$,

2. $\partial_{i}(f) \in \mathcal{J}_{\Gamma}^{k+i}$,

3. if $z \cdot f(\tau, z)$ is holomorphic on $\mathcal{H} \times\{0\}$, also $\partial_{i}^{0}(f) \in \mathcal{J}_{\Gamma}^{k+i}$.

Proof. The proof will use Lemma 3.22 below. To show property (i) of Definition 3.19 first note that by Lemma 3.22 all functions $\iota_{r}(f), \partial_{i}(f)$ and $\partial_{i}^{0}(f)$ stay fixed under the action of $\mid[X]$ for any $X \in L$. From Lemma 3.22 also follows that $\iota_{r}(f), \partial_{i}(f)$ and $\partial_{i}^{0}(f)$ stay fixed under the action of $\left|[A]_{k},\right|[A]_{k+i}$ and $\mid[A]_{k+i}$, respectively, for any $A \in \Gamma$. Thus they transform like Jacobi forms of weight $k, k+i$ and $k+i$, respectively, and index 0 .

For property (ii) note that for any fixed $\tau \in \mathcal{H}$ the set of poles of $\iota_{r}(f), \partial_{i}(f)$ and $\partial_{i}^{0}(f)$ is contained in the $\mathbb{Q}$-span of the set of poles of $f$. This shows property (ii). 
Finally we show property (iii). Again by Lemma 3.22 for any $A \in S L_{2}(\mathbb{Z})$ the functions $\left(\iota_{r}(f)\right)\left|[A]_{k},\left(\partial_{i}(f)\right)\right|[A]_{k+i}$ and $\left(\partial_{i}^{0}(f)\right) \mid[A]_{k+i}$ are equal to $\iota_{r}\left(f \mid[A]_{k}\right)$, $\partial_{i}\left(f \mid[A]_{k}\right)$ and $\partial_{i}^{0}\left(f \mid[A]_{k}\right)$, respectively. Since the action of $\iota_{r}, \partial_{i}$ and $\partial_{i}^{0}$ restricts to the image of $\mathcal{E}_{N}$ (for $\partial_{i}^{0}$ one has to restrict to the subspace on which $\partial_{i}^{0}$ is defined) the functions $\left(\iota_{r}(f)\right)\left|[A]_{k},\left(\partial_{i}(f)\right)\right|[A]_{k+i}$ and $\left(\partial_{i}^{0}(f)\right) \mid[A]_{k+i}$ have property (iii) of Definition 3.19.

Lemma 3.22. Let $f(\tau, z)$ and $g(\tau, z)$ be meromorphic functions on $\mathcal{H} \times \mathbb{C}$, let $X \in L$ and let $A \in S L_{2}(\mathbb{Z})$. Then:

1. $f\left|[A]_{k} \cdot g\right|[A]_{l}=(f g) \mid[A]_{k+l}$,

2. $\left(\iota_{r}(f)\right) \mid[A]_{k}=\iota_{r}\left(f \mid[A]_{k}\right)$ and $\left(\iota_{r}(f)\right) \mid[X]=\iota_{r}(f \mid[r X])$,

3. $\left(\partial_{i}(f)\right) \mid[A]_{k+i}=\partial_{i}\left(f \mid[A]_{k}\right)$,

4. if $z \cdot f(\tau, z)$ is holomorphic on $\mathcal{H} \times\{0\}$, then $\left(\partial_{i}^{0}(f)\right) \mid[A]_{k+i}=\partial_{i}^{0}\left(f \mid[A]_{k}\right)$,

5. $\left(\partial_{i}(f)\right) \mid[X]=\partial_{i}(f \mid[X])$,

6. if $z \cdot f(\tau, z)$ is holomorphic on $\mathcal{H} \times\{0\}$ and $f \mid[X]=f$, then $\left(\partial_{i}^{0}(f)\right) \mid[X]=$ $\partial_{i}^{0}(f \mid[X])$.

Proof. The first two statements follow directly from the definition of the action. Now let $F(\tau, z)$ be any meromorphic function on $\mathcal{H} \times \mathbb{C}$. Then

$$
\begin{aligned}
& \partial_{1}(F)\left|[A]_{k+1}(\tau, z)=\frac{\partial F}{\partial z}\right|[A]_{k+1}(\tau, z)=\frac{\partial F}{\partial z}\left(\frac{a \tau+b}{c \tau+d}, \frac{z}{c \tau+d}\right) \cdot(c \tau+d)^{-(k+1)} \\
& =\frac{\partial}{\partial z}\left(F\left(\frac{a \tau+b}{c \tau+d}, \frac{z}{c \tau+d}\right)\right) \cdot(c \tau+d)^{-k}=\frac{\partial}{\partial z}\left(F \mid[A]_{k}(\tau, z)\right)=\partial_{1}\left(F \mid[A]_{k}\right)(\tau, z) .
\end{aligned}
$$

Thus $\left(\mid[A]_{k+1} \circ \partial_{1}\right)(F)=\left(\partial_{1} \circ \mid[A]_{k}\right)(F)$ for every $F \in \mathcal{M}(\mathcal{H} \times \mathbb{C})$. By induction this implies

$$
\left(\mid[A]_{k+i} \circ \partial_{i}\right)(F)=\left(\partial_{i} \circ \mid[A]_{k}\right)(F)
$$

for every $i \in \mathbb{N}$ and every $F \in \mathcal{M}(\mathcal{H} \times \mathbb{C})$. Now the third statement follows from the last identity by taking $F=f$ and the fourth statement follows by taking $F=z f$ and specializing at $z=0$. Statement (5) follows from the chain rule and statement (6) follows directly from the definitions.

3.4. Proof of the Rigidity Theorem. In this section we show that the elliptic genera $\varphi_{S^{1}}(M)$ and $\varphi_{S^{1}}^{0}(M)$ converge to special Jacobi functions $\psi_{M, R}$ and $\psi_{M, R^{0}}$ of index 0 for $\Gamma_{0}(2)$. We determine the orbit of $\psi_{M, R}$ under the action of the full group $S L_{2}(\mathbb{Z})$ (see Proposition 3.26). Using Corollary 3.17 and the transformation properties of special Jacobi functions we conclude that $\psi_{M, R}$ is holomorphic which finally implies the rigidity theorem for elliptic genera (see Theorem 3.29).

First we want to show that the building blocks defining elliptic genera converge to special Jacobi functions.

It follows from Lemma 3.15 that the $q$-power series $f_{\phi} \in \mathbb{C}\left(\lambda^{\frac{1}{2}}\right)[[q]]$ defined by

$$
f_{\phi}(\lambda)=\left(\lambda^{\frac{1}{2}}-\lambda^{-\frac{1}{2}}\right) \prod_{n=1}^{\infty} \frac{\left(1-q^{n} \lambda\right)\left(1-q^{n} \lambda^{-1}\right)}{\left(1-q^{n}\right)^{2}}
$$

converges normally to a holomorphic function $\phi(q, \lambda)$ on $B \times \widetilde{\mathbb{C}}^{*}$. By Definition 3.1 and Definition 3.7 this means $f_{\phi} \in \mathcal{R}$ and $\phi=\kappa\left(f_{\phi}\right)$. 
Definition 3.23. The Weierstrass' $\boldsymbol{\Phi}$-function is the holomorphic function on $\mathcal{H} \times \mathbb{C}$ defined by

$$
\Phi(\tau, z):=\mathcal{E}_{1}(\phi(q, \lambda))=\phi\left(e^{2 \pi i \cdot \tau}, e^{z}\right) .
$$

For the proof of the following proposition we refer to [HiBeJu92], I.5.

Proposition 3.24. The Weierstrass' $\Phi$-function satisfies the following properties:

1. For fixed $\tau \in \mathcal{H}$ the divisor of $\Phi$ is equal to $2 \pi i(\mathbb{Z} \cdot \tau+\mathbb{Z})$, i.e. $\Phi$ as a function in $z$ has a zero of order one in each point of $2 \pi i(\mathbb{Z} \cdot \tau+\mathbb{Z})$.

2. For any $A=\left(\begin{array}{ll}a & b \\ c & d\end{array}\right) \in S L_{2}(\mathbb{Z})$

$$
\Phi \mid[A]_{-1}(\tau, z)=e^{\frac{c z^{2}}{4 \pi i(c \tau+d)}} \cdot \Phi(\tau, z) .
$$

3. For any $(\alpha, \beta) \in \mathbb{Z} \times \mathbb{Z}$

$$
\Phi(\tau, z+2 \pi i \alpha \tau+2 \pi i \beta)=(-1)^{\alpha+\beta} \cdot e^{-\left(\pi i \alpha^{2} \cdot \tau+\alpha z\right)} \cdot \Phi(\tau, z) .
$$

Recall from Proposition 3.16 that $(\lambda-1) f_{R}(\lambda)$ and $(\lambda-1) f_{R^{0}}(\lambda)$ converge normally on the open set $B_{1}=\left\{(q, \lambda) \in B \times\left.\widetilde{\mathbb{C}}^{*}|| q|<| \lambda|<| q\right|^{-1}\right\}$, especially $f_{R}, f_{R^{0}} \in \mathcal{R}$. In Definition 3.13 the meromorphic functions to which these power series converge were denoted by $\psi_{R}$ and $\psi_{R^{0}}$, i.e. $\psi_{R}=\kappa\left(f_{R}\right)$ and $\psi_{R^{0}}=\kappa\left(f_{R^{0}}\right)$. Let $\Psi_{R}(\tau, z)$ and $\Psi_{R^{0}}(\tau, z)$ be the meromorphic function on $\mathcal{H} \times \mathbb{C}$ defined by

$$
\Psi_{R}(\tau, z):=2 \frac{\Phi(\tau, z+\pi i)}{\Phi(\tau, z) \Phi(\tau, \pi i)} \quad \text { and } \quad \Psi_{R^{0}}(\tau, z):=e^{-z / 2} \cdot \frac{\Phi(2 \tau, z-2 \pi i \cdot \tau)}{\Phi(2 \tau, z) \Phi(2 \tau,-2 \pi i \cdot \tau)} .
$$

Proposition 3.25. Let $\Gamma_{0}(2)$ be the subgroup of $S L_{2}(\mathbb{Z})$ consisting of the matrices $\left(\begin{array}{ll}a & b \\ c & d\end{array}\right)$ with $c \equiv 0 \bmod 2$. Then:

1. The function $\mathcal{E}_{1}\left(\psi_{R}(q, \lambda)\right)$ extends to the meromorphic function $\Psi_{R}(\tau, z)$ on $\mathcal{H} \times \mathbb{C}$, i.e. $\psi_{R}(q, \lambda)$ is equal to $\Psi_{R}(\tau, z)$ for $(q, \lambda)=\left(e^{2 \pi i \tau}, e^{z}\right)$.

2. The function $\mathcal{E}_{1}\left(\psi_{R^{0}}(q, \lambda)\right)$ extends to the meromorphic function $\Psi_{R^{0}}(\tau, z)$ on $\mathcal{H} \times \mathbb{C}$, i.e. $\psi_{R^{0}}(q, \lambda)$ is equal to $\Psi_{R^{0}}(\tau, z)$ for $(q, \lambda)=\left(e^{2 \pi i \tau}, e^{z}\right)$.

3. The functions $\Psi_{R}(\tau, z)$ and $\Psi_{R^{0}}(\tau, z)$ on $\mathcal{H} \times \mathbb{C}$ are special Jacobi functions in $\mathcal{J}_{\Gamma_{0}(2)}^{1}$.

4. The orbit of $\Psi_{R}(\tau, z)$ under the action of $S L_{2}(\mathbb{Z})$ given by $f \mapsto f \mid[A]_{1}$ is equal to

$$
\left\{\Psi_{R}(\tau, z), 2 \Psi_{R^{0}}(\tau / 2, z), 2 \Psi_{R^{0}}((\tau+1) / 2, z)\right\},
$$

i.e. the orbit consists of the meromorphic extensions of

$$
\left\{\psi_{R}(q, \lambda), 2 \psi_{R^{0}}\left(q^{\frac{1}{2}}, \lambda\right), 2 \psi_{R^{0}}\left(-q^{\frac{1}{2}}, \lambda\right)\right\}
$$

for $(q, \lambda)=\left(e^{2 \pi i \tau}, e^{z}\right)$.

Proof. From the definition of the Weierstrass' $\Phi$-function it follows that $\Phi(\tau, z+\pi i)$ is defined by the $q$-power series $i\left(\lambda^{\frac{1}{2}}+\lambda^{-\frac{1}{2}}\right) \prod_{n=1}^{\infty} \frac{\left(1+q^{n} \lambda\right)\left(1+q^{n} \lambda^{-1}\right)}{\left(1-q^{n}\right)^{2}}=f_{\phi}(-\lambda)$ and $\Phi(\tau, \pi i)$ is defined by $2 i \prod_{n=1}^{\infty} \frac{\left(1+q^{n}\right)^{2}}{\left(1-q^{n}\right)^{2}}=f_{\phi}(-1)$ for $(q, \lambda)=\left(e^{2 \pi i \cdot \tau}, e^{z}\right)$. More precisely $\Phi(\tau, z+\pi i)=\mathcal{E}_{1}\left(\kappa\left(f_{\phi}(-\lambda)\right)\right)$ and $\Phi(\tau, \pi i)=\mathcal{E}_{1}\left(\kappa\left(f_{\phi}(-1)\right)\right)$. Thus

$$
\begin{aligned}
\Psi_{R}(\tau, z) & =2 \frac{\Phi(\tau, z+\pi i)}{\Phi(\tau, z) \Phi(\tau, \pi i)}=\mathcal{E}_{1}\left(\kappa\left(\frac{f_{\phi}(-\lambda)}{f_{\phi}(\lambda) \cdot f_{\phi}(-1)}\right)\right) \\
& =\mathcal{E}_{1}\left(\kappa\left(f_{R}(\lambda)\right)\right)=\mathcal{E}_{1}\left(\psi_{R}(q, \lambda)\right),
\end{aligned}
$$


so $\Psi_{R}(\tau, z)$ is equal to $\psi_{R}(q, \lambda)$ for $(q, \lambda)=\left(e^{2 \pi i \cdot \tau}, e^{z}\right)$. The proof of the second part is similar.

To show Part 3 we use Proposition 3.24. From the divisor property of $\Phi$ it follows directly that for fixed $\tau$ the poles of $\Psi_{R}(\tau, z)$ and $\Psi_{R^{0}}(\tau, z)$ are contained in $2 \pi i(\mathbb{Q} \cdot \tau+\mathbb{Q})$. From Part 1 and 2 it follows that $\Psi_{R}(\tau, z)$ and $\Psi_{R^{0}}(\tau, z)$ are in the image of $\mathcal{E}_{1}$. So we are left to show that both functions transform like Jacobi forms of weight 1 and index 0 for $\Gamma_{0}(2)$.

First let $X=(\alpha, \beta)$ be any element of $L$ (note that $\alpha$ and $\beta$ are even). From Proposition 3.24, Part 3, it follows easily

$$
\Psi_{R}(\tau, z) \mid[X]=\Psi_{R}(\tau, z) \quad \text { and } \quad \Psi_{R^{0}}(\tau, z) \mid[X]=\Psi_{R^{0}}(\tau, z) .
$$

Next we want to show that both functions are invariant under $\mid[A]_{1}$ for any $A \in$ $\Gamma_{0}(2)$. Let $S:=\left(\begin{array}{rr}0 & 1 \\ -1 & 0\end{array}\right)$ and $T:=\left(\begin{array}{ll}1 & 1 \\ 0 & 1\end{array}\right)$. Note that $\Gamma_{0}(2)$ is generated by $T$ and $S T^{2} S$. It follows directly that $\Psi_{R}(\tau, z)$ and $\Psi_{R^{0}}(\tau, z)$ are fixed under $\mid[T]_{1}$. Furthermore a straightforward calculation using Proposition 3.24, Part 2, shows that the operation $\mid[S]_{1}$ interchanges $\Psi_{R}(\tau, z)$ and $2 \Psi_{R^{0}}(\tau / 2, z)$ and also interchanges $\Psi_{R^{0}}(\tau, z)$ and $\frac{1}{2} \Psi_{R}(\tau / 2, z / 2)$. From Proposition 3.24, Part 2, it follows also that $\Psi_{R^{0}}(\tau / 2, z)$ and $\Psi_{R}(\tau / 2, z / 2)$ are fixed under $\mid\left[T^{2}\right]_{1}$. Thus $\Psi_{R}(\tau, z)$ and $\Psi_{R^{0}}(\tau, z)$ are fixed under $\Gamma_{0}(2)=\left\langle T, S T^{2} S\right\rangle$. This finishes the proof of the third part.

For the last part note that $\Gamma_{0}(2)$ has index 3 in $S L_{2}(\mathbb{Z})$ and coset representatives $I d, S$ and $S T, \Psi_{R}(\tau, z)$ is fixed under the action of $\Gamma_{0}(2)$ and $\Psi_{R}(\tau, z) \mid[S]_{1}=$ $2 \Psi_{R^{0}}(\tau / 2, z)$.

Proposition 3.26. Let $R, R^{0} \in R(\operatorname{Spin}(2 m))[[q]]$ be the exponential representations defining elliptic genera. Let $M$ be a $2 m$-dimensional Spin-manifold with Spin-preserving $S^{1}$-action.

1. The functions $\psi_{M, R}=\kappa\left(\varphi_{S^{1}}(M)\right)$ and $\psi_{M, R^{0}}=\kappa\left(\varphi_{S^{1}}^{0}(M)\right)$ are in $\mathcal{J}_{\Gamma_{0}(2)}^{m}$ for $(q, \lambda)=\left(e^{2 \pi i \tau}, e^{z}\right)$.

2. The orbit of $\psi_{M, R}$ under the action of $S L_{2}(\mathbb{Z})$ is given by

$$
\left\{\psi_{M, R}(q, \lambda), 2^{m} \psi_{M, R^{0}}\left(q^{\frac{1}{2}}, \lambda\right), 2^{m} \psi_{M, R^{0}}\left(-q^{\frac{1}{2}}, \lambda\right)\right\} .
$$

Proof. Substituting $\left(e^{2 \pi i \tau}, e^{z}\right)$ for $(q, \lambda)$ means applying the map $\mathcal{E}_{1}$. Now

$$
\mathcal{E}_{1}\left(\psi_{M, R}\right)=\mathcal{E}_{1}\left(\mathfrak{P}_{M}\left(\psi_{R}\right)\right)=\mathfrak{P}_{M}\left(\mathcal{E}_{1}\left(\psi_{R}\right)\right)=\mathfrak{P}_{M}\left(\Psi_{R}\right) .
$$

The first equation is due to Corollary 3.17, Part 3, the second is the fact that $\mathcal{E}_{1}$ and $\mathfrak{P}_{M}$ commute, and the last one is Proposition 3.25, Part 1. By Part 3 of the same proposition, $\Psi_{R}$ is in $\mathcal{J}_{\Gamma_{0}(2)}^{1}$. Since $\mathcal{J}_{\Gamma_{0}(2)}^{*}$ is a DSC-ring of functions we get by Lemma $3.6 \mathcal{E}_{1}\left(\psi_{M, R}\right)=\mathfrak{P}_{M}\left(\Psi_{R}\right) \in \mathcal{J}_{\Gamma_{0}(2)}^{m}$. The argument for $\psi_{M, R^{0}}$ is analogous.

For the second statement note that from the above and Lemma 3.22 we get

$$
\left(\mathcal{E}_{1}\left(\psi_{M, R}\right)\right) \mid[A]_{m}=\mathfrak{P}_{M}\left(\Psi_{R} \mid[A]_{1}\right) .
$$

So the orbit is given by Proposition 3.25, Part 4.

The proposition gives a more precise meaning to the statement that the elliptic genera $\varphi_{S^{1}}(M)$ and $\varphi_{S^{1}}^{0}(M)$ are two descriptions of the same invariant of the manifold $M$. This invariant is a special Jacobi function. The genera are essentially Fourier expansions of the orbit of this invariant under the $S L_{2}(\mathbb{Z})$-action.

To finally prove the rigidity theorem we will use a corollary to the following 
Proposition 3.27. Let $f(\tau, z)$ be a meromorphic function on $\mathcal{H} \times \mathbb{C}$, s.t. $f$ has only poles for $z \in 2 \pi i \cdot(\mathbb{Q} \cdot \tau+\mathbb{Q})$ and for all $A \in S L_{2}(\mathbb{Z})$ the function $f \mid[A]_{k}$ has no poles for $z \in i \mathbb{R}$. Then for any fixed $\tau \in \mathcal{H}$ the function $f$ and all the functions $f \mid[A]_{k}$ are holomorphic as functions in $z \in \mathbb{C}$.

Proof. It suffices to prove the statement for $f$. By the definition of the action of a matrix $A=\left(\begin{array}{ll}a & b \\ c & d\end{array}\right) \in S L_{2}(\mathbb{Z})$ on $\mathcal{M}(\mathcal{H} \times \mathbb{C})$ we have

$$
f(\tau, 2 \pi i(\alpha \tau+\beta))=\left(c \tau^{\prime}+d\right)^{k}\left(f \mid[A]_{k}\right)\left(\tau^{\prime}, 2 \pi i\left(\alpha^{\prime} \tau^{\prime}+\beta^{\prime}\right)\right),
$$

where $\tau^{\prime}=A^{-1} \tau$ and $\left(\alpha^{\prime}, \beta^{\prime}\right)=(\alpha, \beta) A$ for $\alpha, \beta \in \mathbb{Q}$ and $\tau \in \mathcal{H}$. If $\tau \in \mathcal{H}$ is fixed and $f$ has a pole in $2 \pi i(\alpha \tau+\beta)$ we can choose a matrix $A \in S L_{2}(\mathbb{Z})$ s.t. $(\alpha, \beta) A=\left(0, \beta^{\prime}\right)$ for some $\beta^{\prime} \in \mathbb{Q}$. Formula (10) now shows that $f \mid[A]_{k}$ must have a pole in $2 \pi i \beta^{\prime} \in i \mathbb{R}$ for fixed $\tau^{\prime}=A^{-1} \tau \in \mathcal{H}$. This contradicts the assumptions. So $f$ has no pole for fixed $\tau$, i.e. it is holomorphic as a function in $z \in \mathbb{C}$.

Corollary 3.28. Let $f(\tau, z) \in \mathcal{J}_{\Gamma}^{k}$ such that for all $A \in S L_{2}(\mathbb{Z})$ the function $f \mid[A]_{k}$ has no poles for $z \in i \mathbb{R}$. Then $f$ and all the functions $f \mid[A]_{k}$ are constant in $z$. Moreover they are holomorphic on $\mathcal{H} \times \mathbb{C}$.

Proof. By Proposition 3.27 for any fixed $\tau \in \mathcal{H}$ the function $f \mid[A]_{k}$ is holomorphic in $z \in \mathbb{C}$. The second transformation property for special Jacobi functions given in Definition 3.18 implies that $f \mid[A]_{k}$ as a function in $z$ is elliptic with respect to the lattice $2 \pi i(2 \mathbb{Z} \cdot \tau+2 \mathbb{Z})$. Since for any fixed $\tau \in \mathcal{H}$ the function $f \mid[A]_{k}$ is holomorphic in $z$ it has to be constant in $z$. Since $f \mid[A]_{k}$ is meromorphic on $\mathcal{H} \times \mathbb{C}$ and is constant in $z$ it is holomorphic on $\mathcal{H} \times \mathbb{C}$.

Theorem 3.29 (Rigidity Theorem). Let $M$ be a $2 m$-dimensional Spin-manifold with Spin-preserving $S^{1}$-action. Then the equivariant elliptic genera $\varphi_{S^{1}}(M)$ and $\varphi_{S^{1}}^{0}(M)$ are both rigid, i.e. they are power series in $q$ with coefficients which are constant as characters of $S^{1}$. In other words

$$
\varphi_{S^{1}}(M)=\varphi(M) \quad \text { and } \quad \varphi_{S^{1}}^{0}(M)=\varphi^{0}(M) .
$$

Proof. In Corollary 3.17 we proved that the equivariant elliptic genera $\varphi_{S^{1}}(M)$ and $\varphi_{S^{1}}^{0}(M)$ converge normally on some $B_{N}$ to holomorphic functions $\psi_{M, R}$ and $\psi_{M, R^{0}}$. In particular both have no poles on $S^{1}$. In Proposition 3.26 we identified these functions as elements in $\mathcal{J}_{\Gamma_{0}(2)}^{m}$. In particular they have only poles for $z \in$ $2 \pi i \cdot(\mathbb{Q} \cdot \tau+\mathbb{Q})$, but still no poles for $\lambda=e^{z}$ in $S^{1}$. Furthermore the orbit of $\psi_{M, R}$ under the action of $S L_{2}(\mathbb{Z})$ is given by the functions $\psi_{M, R}(q, \lambda), 2^{m} \psi_{M, R^{0}}\left(q^{\frac{1}{2}}, \lambda\right)$, and $2^{m} \psi_{M, R^{0}}\left(-q^{\frac{1}{2}}, \lambda\right)$, all of which have no poles for $\lambda=e^{z} \in S^{1}$. But then Corollary 3.28 shows that $\psi_{M, R}$ (and also $\psi_{M, R^{0}}$ ) is constant in $\lambda$. So from the normal convergence of the equivariant elliptic genera $\varphi_{S^{1}}(M)$ and $\varphi_{S^{1}}^{0}(M)$ to the limit functions $\psi_{M, R}$ and $\psi_{M, R^{0}}$, it follows that the equivariant elliptic genera $\varphi_{S^{1}}(M)$ and $\varphi_{S^{1}}^{0}(M)$ themselves are constant in $\lambda$.

\section{ACKNOWLEDGEMENTS}

We wish to thank S. Ochanine for drawing our attention to the problem, Th. Berger for numerous discussions and M. Kreck for his encouragement. We also would like to thank the referee for valuable suggestions. 


\section{REFERENCES}

[Ah66] L.V. Ahlfors: Complex Analysis (2nd Edition), International Series in Pure and Applied Mathematics, McGraw-Hill (1966). MR 32:5844

[AtSiIII68] M.F. Atiyah and I.M. Singer, The index of elliptic operators: III, Ann. of Math. 87 (1968), 546-604. MR 38:5245

[Ca63] H. Cartan: Elementary theory of analytic functions of one or several complex variables, Addison-Wesley (1963). MR 27:4911

[BoTa89] R. Bott and C.H. Taubes: On the rigidity theorems of Witten, J. Amer. Math. Soc. 2 (1989), 137-186. MR 89k:58270

[De96] A. Dessai: Rigidity Theorems for Spin ${ }^{c}$-Manifolds and Applications, doctoral thesis, Universität Mainz (1996).

[EiZa85] M. Eichler and D. Zagier: The Theory of Jacobi Forms, Progress in Mathematics, Vol 55, Birkhäuser (1985). MR 86j:11043

[Hi88] F. Hirzebruch: Elliptic genera of level $N$ for complex manifolds, in: K. Bleuler and M. Werner (Eds.): Differential Geometrical Methods in Theoretical Physics (Como 1987), NATO Adv. Sci. Inst. Ser. C: Math. Phys. Sci., 250, Kluwer (1988). MR 90m:57030

[HiBeJu92] F. Hirzebruch, Th. Berger and R. Jung: Manifolds and Modular Forms, Aspects of Mathematics, Vol. E20, Vieweg (1992). MR 94d:57001

[La86] P.S. Landweber: Elliptic Genera: an Introductory Overview, in [La88], 1-10. CMP 21:04

[La88] P.S. Landweber (Ed.): Elliptic Curves and Modular Forms in Algebraic Topology, Proceedings Princeton 1986, Lecture Notes in Mathematics, 1326, Springer (1988). MR 91a:57021

[LaSt88] P.S. Landweber and R.E. Stong: Circle Actions on Spin Manifolds and Characteristic Numbers, Topology 27 (1988), 145-161. MR 90a:57040

[Li92] K. Liu, On Elliptic Genera, Theta-Functions and Elliptic Modular Surfaces, preprint (1992).

[Li95] $\quad$ On modular invariance and rigidity theorems, J. Differential Geom. 41 (1995), 343-396. MR 96f:58152

[Oc86] S. Ochanine: Genres Elliptiques Équivariants, in [La88], 107-122. CMP 21:04

[Oc87] _ Sur les genres multiplicatifs définis par des intégrales elliptiques, Topology 26 (1987), 143-151. MR 88e:57031

[Re90] R. Remmert: Theory of Complex Functions, Graduate Texts in Mathematics, 122, Springer (1990). MR 91m:30001

[Ta89] C.H. Taubes: $S^{1}$ Actions and Elliptic Genera, Comm. Math. Phys. 122 (1989), 455526. MR 90f:58167

[Wi86] E. Witten: The Index of the Dirac Operator in Loop Space, in [La88], 161-181. CMP 21:04

Department of Mathematics, University of Mainz, 55099 Mainz, Germany

Current address: Department of Mathematics, University of Augsburg, 86135 Augsburg, Germany

E-mail address: dessai@mathpool.Uni-Augsburg.DE

URL: http://www.math.Uni-Augsburg.DE/geo/dessai/homepage.html

Mathematisches Forschungsinstitut Oberwolfach, Lorenzenhof, 77709 Oberwolfach, Germany

E-mail address: jung@MFO.DE 